\title{
Feynman rules for effective Regge action
}

\author{
E.N. Antonov ${ }^{a}$, I.O. Cherednikov ${ }^{\text {b,c }}$, E.A. Kuraev ${ }^{\text {b }}$, L.N. Lipatov ${ }^{\text {a,d }}$ \\ ${ }^{a}$ Petersburg Nuclear Physics Institute, RU-188350 PNPI, Gatchina, St. Petersburg, Russia \\ b Joint Institute for Nuclear Research, RU-141980 BLTP JINR, Dubna, Russia \\ ${ }^{\mathrm{c}}$ Dipartimento di Fisica, Università di Cagliari and INFN-Cagliari, C.P. 170, I-90142 Monserrato (CA), Italy \\ ${ }^{\mathrm{d}}$ Laboratoire de Physique Theorique et Hautes Energies, Université Pierre et Marie Curie, \\ BP 126, 4, place Jussieu, F-75252 Paris Cedex 05, France
}

Received 11 March 2005; received in revised form 17 May 2005; accepted 24 May 2005

Available online 13 June 2005

\begin{abstract}
Starting from the gauge invariant effective action in the quasi-multi-Regge kinematics (QMRK), we obtain the effective reggeized gluon $(\mathrm{R})$-particle $(\mathrm{P})$ vertices of the following types: $R P P, R R P$, $R R P P, R P P P, R R P P P$, and $R P P P P$, where the on-mass-shell particles are gluons, or sets of gluons with small invariant masses. The explicit expressions satisfying the Bose-symmetry and gauge invariance conditions are obtained. As a comment to the Feynman rules for derivation of the amplitudes in terms of effective vertices we present a "vocabulary" for practitioners.
\end{abstract}

(c) 2005 Elsevier B.V. All rights reserved.

PACS: 12.38.-t; $12.40 . \mathrm{Nn}$

\section{Introduction}

The unitarization of the BFKL Pomeron as a composite state of two reggeized gluons is among the most actual problems for investigations in QCD [1]. The BFKL evolution is applied to describe the hadronic scattering amplitudes in the Regge regime $s \rightarrow \infty$, $s \gg-t$ and the high-energy behavior of total cross sections for semi-hard QCD processes at $Q^{2} \gg \Lambda_{\mathrm{QCD}}^{2}$. In particular, the BFKL equation together with the DGLAP equation [2]

E-mail addresses: antonov@thd.pnpi.spb.ru (E.N. Antonov), igor.cherednikov@jinr.ru (I.O. Cherednikov), kuraev@thsun1.jinr.ru (E.A. Kuraev), lipatov@thd.pnpi.spb.ru (L.N. Lipatov). 
are used to sum up the logarithmic contributions to structure functions in the deep inelastic $e p$ scattering, and they are relevant to the gluon saturation at small Bjorken variable $x_{B j}-$ in particular, in connection with the rapid growth of the function $F_{2}\left(x, Q^{2}\right)$, observed at HERA [3].

In the leading logarithmic approximation (LLA), the BFKL equation predicts a powerlike rise of cross sections with energy, which obviously violates the Froissart constraint $\sigma_{t}<c \ln ^{2} s$ being a consequence of the $s$-channel unitarity. One possible method to overcome this difficulty is to take into account the multiple Pomeron exchanges in the eikonal approximation [4]. A more consistent approach is based on the solution of the BKP equation which is a generalization of the BFKL equation for the case of composite states of several reggeized gluons [5]. For large $N_{c}$, the BKP equation turns out to be integrable [6]. For the unitarization purpose, it is possible also to use an effective Lagrangian written for the multi-Regge kinematics of gluons in intermediate states of the direct $s$ - and $u$-channels [7]. In this kinematics, the Feynman diagrams are factorized in the product of integrals over longitudinal and transverse subspaces. But the most general approach consists in reformulation of QCD in terms of a gauge-invariant effective field theory for the reggeized gluon interactions $[8,9]$. Below we develop this approach and apply it to the problem of calculating production amplitudes in the quasi-multi-Regge kinematics (QMRK) for the final particles.

The high-energy scattering amplitudes can be presented in terms of the Wilson $\mathcal{P}$ exponentials [10]. For a quasi-elastic kinematics, one can derive a two-dimensional sigmamodel defined in the transverse subspace and constructed in terms of these $\mathcal{P}$-exponentials [11]. However, this approach is incomplete and suffers from some shortcomings (see [8, 12]). In the works by Balitsky [13], the high-energy scattering was considered by means of the amplitude factorization in the rapidity space within the shock-wave picture, and an hierarchy of equations for scattering amplitudes was derived. The similar equations were obtained in the framework of the JIMWLK approach [14]. In these models, the highenergy behavior in the large $N_{c}$ limit is described by a non-linear evolution equation, now known as the Balitsky-Kovchegov (BK) equation [13,15]. It has a simple interpretation in the framework of the dipole picture [16]. In turns, the dipole picture corresponds to the Möbius representation for the Pomeron wave function, Ref. [17]. The similar, to some extent, methods based on the renormalization group arguments were developed in Ref. [18] (see also [19]).

On the other hand, the straightforward way to deal with the unitarization problem is to evaluate non-leading contributions to the BFKL equation. The explicit calculation of the NLO logarithmic corrections to inelastic amplitudes was performed in Ref. [20] for one and two gluon production. Later on, the full result for the integral kernel of the BFKL equation in the next-to-leading approximation was obtained [21,22]. Although the corrections are large, the modified equation can be used in the phenomenological applications (see, for example, Refs. $[23,25,26])$.

In the present study, we use the effective action approach proposed in Ref. [8]. It appears to be convenient and physically well-motivated to perform the systematic self-consistent calculation of non-leading contributions to the BFKL Pomeron intercept by means of an effective theory describing the interactions of reggeized gluons with elementary particles (quarks and gluons). This approach describes the processes with an arbitrary number 
of clusters of real and virtual particles separated by large rapidity gaps, corresponding to QMRK for the produced particles. For interactions local in the relative rapidities of particles the non-Abelian gauge invariant effective action was derived [8]. This action contains the fields of two sorts: the fields $A_{ \pm}$, describing the reggeized gluons living in the $t$-channel, and the ordinary gluons and quarks produced and annihilated in the direct channels.

The motivation of this work is the necessity of developing a proper practical tool for explicit calculations of the next-to-leading corrections to the Feynman inelastic amplitudes of multi-peripheral processes. The first thing one has to do before doing any calculations is to derive the Feynman rules corresponding to an effective Lagrangian which is dealt with. In our case, such rules should prescribe the propagators of reggeons and ordinary particles as well as the effective reggeon-particle vertices. A number of such effective vertices was already constructed [1,8,9,24] (see also [27]). Note, that in Ref. [20] for the construction of production amplitudes the authors used the string theory motivated approach [28].

Below we reproduce the known results and calculate new effective vertices within the unified framework of the gauge invariant effective action suggested in Ref. [8]. There is a hope that the formulation of the Feynman rules derived from the effective action can provide a significant simplification for construction of the differential cross sections describing the creation of several clusters of particles separated by rapidity gaps. We concentrate our efforts on the presentation of the results in the form convenient for their use in numerical simulations and in phenomenological applications.

The processes of such a kind can be investigated, e.g., in experiments at RHIC and LHC. Another important branch of possible applications - calculation of peripheral amplitudes with several reggeized gluons in the crossing channel-is out of scope of the present paper and will not be considered here.

The structure of the paper is following: in Section 2, we fix notations, give an elementary "vocabulary" of terms and kinematic constraints that are used in the work and remind the basic relations for the effective action approach of Ref. [8]. In Section 3, several reggeonparticle amplitudes are derived systematically within this approach and presented in an explicit form. In Section 4, we discuss the calculation of the cross sections for the multijet production process in the QMRK using the effective vertices derived in this paper. In conclusions, we summarize the results and propose possible applications of them in highenergy phenomenology.

Some technical details are given in Appendix A.

\section{Effective action and recurrence relations for the reggeon vertices}

We consider the parton-parton collision at high energies $\sqrt{s}$ in the center-of-mass system. The main contribution to the total cross section stems from QMRK of final state particles (see Fig. 1). In this regime, the final state particles compose several groups (clusters) with an arbitrary number of gluons or/and quarks with a fixed mass $M_{i}(i=1,2, \ldots, n)$ of each group. The clusters are produced in the multi-Regge kinematics with respect to each other:

$$
P_{A}+P_{B}=Q_{1}+Q_{2}+\cdots+Q_{n},
$$




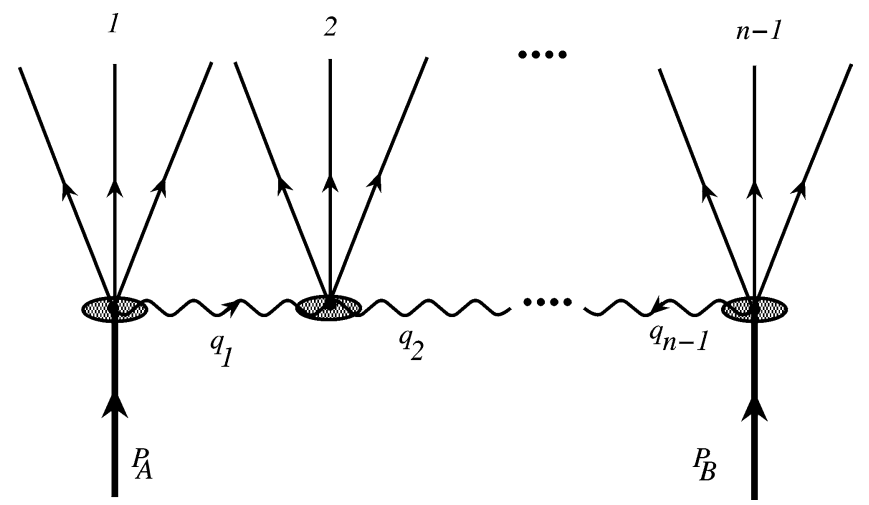

Fig. 1. Quasi-multi-Regge kinematics: notations. Process of $n$-jet production $2 \rightarrow n_{\text {jets }}$ in the quasi-multi-Regge kinematics.

$$
\begin{aligned}
& s=2 P_{A} P_{B}=4 E^{2} \gg s_{i}=2 Q_{i} Q_{i+1} \gg\left|t_{i}\right|=\left|q_{i}^{2}\right|, \quad i=1,2, \ldots, n-1 ; \\
& Q_{i}^{2}=M_{i}^{2}, \quad Q_{k}=\sum_{j} p_{j}^{(k)}, \quad k=1,2, \ldots, n .
\end{aligned}
$$

Also, we introduce the light-cone vectors

$$
n^{+}=\frac{P_{B}}{E}, \quad n^{-}=\frac{P_{A}}{E}, \quad n^{+} n^{-}=2, \quad\left(n^{ \pm}\right)^{2}=0
$$

and thus the light-cone projections of momenta and derivatives read, respectively

$$
k^{ \pm}=\left(n^{ \pm}\right)_{\mu} \cdot k^{\mu}, \quad \partial_{ \pm}=\left(n^{ \pm}\right)_{\mu} \cdot \partial^{\mu} .
$$

We imply also that the derivatives act on the particle $v(p)$ and reggeon $A(p)$ fields in the momentum representation as follows:

$$
\begin{aligned}
& \partial_{ \pm} v(p)=-i p^{ \pm} v(p), \quad \frac{1}{\partial_{ \pm}} v(p)=\frac{i}{p^{ \pm}} v(p), \\
& \partial_{\sigma}^{2} A(q)=-q^{2} A(q), \quad q^{2}=-q_{\perp}^{2} .
\end{aligned}
$$

In the particular case, when there are only 3 clusters in the final states (see Fig. 1), we have two momentum transfers:

$$
q_{1}=P_{A}-P_{A^{\prime}}, \quad q_{2}=P_{B}-P_{B^{\prime}}
$$

with their Sudakov decompositions

$$
q_{1}=\boldsymbol{q}_{1 \perp}+\frac{q_{1}^{+}}{2} n^{-}, \quad q_{2}=\boldsymbol{q}_{2 \perp}+\frac{q_{2}^{-}}{2} n^{+}, \quad q_{1}^{-}=q_{2}^{+}=0 .
$$

The Sudakov variables for produced particles are:

$$
p_{i}=\frac{p_{i}^{+}}{2} n^{-}+\frac{p_{i}^{-}}{2} n^{+}+\boldsymbol{p}_{i \perp} .
$$


In the fragmentation regions, one has

$$
\begin{aligned}
& P_{A}+q_{2} \rightarrow p_{1}+p_{2}+\cdots+p_{n}: \quad P_{A}^{+}=\sum_{i=1}^{n} p_{i}^{+}, \quad\left|q_{2}^{+}\right| \ll p_{i}^{+}, \\
& P_{B}+q_{1} \rightarrow p_{1}+p_{2}+\cdots+p_{n}:
\end{aligned}
$$

In the central region one has

$$
q_{1}+q_{2} \rightarrow \sum_{i=1}^{n} p_{i}, \quad q_{1}^{+}=\sum_{i=1}^{n} p_{i}^{+} ; \quad q_{2}^{-}=\sum_{i=1}^{n} p_{i}^{-} .
$$

To each reggeized gluon line (drawn in the crossing channels $t_{i}$ in the Fig. 1), one should attribute the sign $(+)$ (production) to its end attached to initial parton with a large Sudakov component along $P_{A}$, and the sign (-) (annihilation) to the end attached to parton with a large Sudakov component along $P_{B}$. Note, that wavy lines in Fig. 1 represent the reggeized gluons which are off-mass-shell particles lying on the Regge trajectory. Initial, final and produced particles (presented by the bold lines in the Fig. 1) are on-mass-shell particles considered to be massless. Let us emphasize that the presence of reggeon lines within any production block is forbidden in QMRK, Eq. (1).

To formulate the effective action one should introduce apart from the usual fields $\psi(x)$, $\bar{\psi}$ and $v_{\mu}(x)$ describing the quarks and gluons, also the fields $A_{ \pm}(x)$ describing the production and annihilation of the reggeized gluons in the crossing channel. The quantities $v_{\mu}(x), A_{ \pm}(x)$ are implied to be anti-Hermitian matrices belonging to the fundamental representation of the $S U(N)$ algebra:

$$
v_{\mu}=-i T^{a} v_{\mu}^{a}, \quad A_{ \pm}=-i T^{a} A_{ \pm}^{a}, \quad\left[T^{a}, T^{b}\right]=i f_{a b c} T^{c}, \operatorname{Tr}\left(T^{a} T^{b}\right)=\frac{1}{2} \delta^{a b} .
$$

Here $T^{a}$ are Hermitian generators of the color group in the fundamental representation.

It is convenient to write down the effective action in the following form [8]:

$$
S=\int d^{4} x\left[\mathcal{L}_{\mathrm{QCD}}+\mathcal{L}_{\text {ind }}\right]
$$

where the standard Yang-Mills part consists of the quark-gluon and gluon-gluon interactions

$$
\mathcal{L}_{\mathrm{QCD}}=i \bar{\psi} \hat{D} \psi+\frac{1}{2} \operatorname{Tr} G_{\mu \nu}^{2}=\mathcal{L}_{k}+\mathcal{L}_{\text {int }}, \quad D_{\mu}=\partial_{\mu}+g v_{\mu}, \quad G_{\mu \nu}=\frac{1}{g}\left[D_{\mu}, D_{\nu}\right] .
$$

The explicit forms of kinetic and interacting parts of the QCD Lagrangian are:

$$
\begin{aligned}
& \mathcal{L}_{k}=i \bar{\psi} \hat{\partial} \psi-\frac{1}{4}\left(\partial_{\mu} v_{v}^{a}-\partial_{\nu} v_{\mu}^{a}\right)^{2} \\
& \mathcal{L}_{\text {int }}=-g \bar{\psi} \hat{v}^{a} \psi+\frac{g}{2} f_{a b c}\left(\partial_{\mu} v_{\nu}^{a}\right) v_{\mu}^{b} v_{v}^{c}-\frac{g^{2}}{4} f_{a b c} f_{a d e} v_{\mu}^{b} v_{\nu}^{c} v_{\mu}^{d} v_{v}^{e} .
\end{aligned}
$$


The induced part

$$
\mathcal{L}_{\text {ind }}=\mathcal{L}_{\text {ind }}^{k}+\mathcal{L}_{\text {ind }}^{\mathrm{GR}}, \quad \mathcal{L}_{\text {ind }}^{k}=-\partial_{\mu} A_{+}^{a} \cdot \partial_{\mu} A_{-}^{a}
$$

contains apart from the kinetic term $\mathcal{L}_{\text {ind }}^{k}$ also gluon-reggeon couplings [8]

$$
\begin{aligned}
\mathcal{L}_{\text {ind }}^{\mathrm{GR}}\left(v_{ \pm}, A_{ \pm}\right)= & -\operatorname{Tr}\left\{\frac{1}{g} \partial_{+}\left[\mathcal{P} \exp \left(-\frac{g}{2} \int_{-\infty}^{x^{+}} v_{+}\left(x^{\prime}\right) d x^{\prime}\right)\right] \cdot \partial_{\sigma}^{2} A_{-}(x)\right. \\
& \left.+\frac{1}{g} \partial_{-}\left[\mathcal{P} \exp \left(-\frac{g}{2} \int_{-\infty}^{x^{-}} v_{-}\left(x^{\prime}\right) d x^{\prime}\right)\right] \cdot \partial_{\sigma}^{2} A_{+}(x)\right\} \\
= & \operatorname{Tr}\left\{\left[v_{+}-g v_{+} \frac{1}{\partial_{+}} v_{+}+g^{2} v_{+} \frac{1}{\partial_{+}} v_{+} \frac{1}{\partial_{+}} v_{+}-\cdots\right] \partial_{\sigma}^{2} A_{-}\right. \\
& \left.+\left[v_{-}-g v_{-} \frac{1}{\partial_{-}} v_{-}+g^{2} v_{-} \frac{1}{\partial_{-}} v_{-} \frac{1}{\partial_{-}} v_{-}-\cdots\right] \partial_{\sigma}^{2} A_{+}\right\},
\end{aligned}
$$

where the symbol $\mathcal{P}$ orders the multiplication of matrices $v_{ \pm}\left(x^{\prime}\right)$ in accordance with increasing of their arguments $x^{\prime \pm}$. Note, that in QMRK momenta of reggeons are transverse $-q_{i}^{2} \approx \boldsymbol{q}_{i}^{2}$ and the following kinematic relation is implied for the fields

$$
\partial_{-} A_{+}=\partial_{+} A_{-}=0
$$

which guarantees the gauge-invariance of the action providing that the fields $A_{ \pm}$are not changed under the local gauge transformations (see Refs. [8,9]). Because $\mathcal{L}_{\text {ind }}^{\mathrm{GR}}$ contains the linear term in $v_{ \pm}$, the Euler-Lagrange equation for the functional $S$ has a non-trivial classical solution $v_{ \pm}=A_{ \pm}+\cdots$ even in the perturbation theory, which leads to a renormalization of the free action for the fields $A_{ \pm}[8,9]$. Instead of the subtraction of $A_{ \pm}$from the gluon field $v_{ \pm}$using the equations of motion, we include in the Feynman rules the vertex for the direct transition between the reggeon and gluon fields. It corresponds to the perturbative solution of the equations of motion.

Going to the momentum space, one can define a sequence of the effective vertices $\Delta_{a_{0} a_{1} \ldots a_{r} c}^{v_{0} v_{1} \ldots v_{r}}\left(k_{0}^{+}, k_{1}^{+}, \ldots, k_{r}^{+}\right)$for the interaction of the field $A_{+}^{c}$ with $(r+1)$ gluons with color indices $a_{0} a_{1} \ldots a_{r}$ and momenta $k_{0}, k_{1}, \ldots, k_{r}$ and corresponding vertices for the interaction of the field $A_{-}^{c}[8,9]$. Note, that for the usual and effective gluon vertices we imply all the momenta to be in-coming. The Sudakov components $k_{l}^{+}$satisfy the conservation law

$$
k_{0}^{+}+k_{1}^{+}+\cdots+k_{r}^{+}=0,
$$

since the component $q^{+}$of the reggeon momentum is negligibly small.

For example, for $r=0,1,2$ we have [8,9]

$$
\begin{aligned}
& \Delta_{a_{0} c}^{v_{0} \pm}=\boldsymbol{q}_{\perp}^{2}\left(n^{ \pm}\right)^{\nu_{0}} \delta^{a_{0} c}, \\
& \Delta_{a_{0} a_{1} c}^{v_{0} \nu_{1}+}\left(k_{0}^{+}, k_{1}^{+}\right)=-i \boldsymbol{q}_{\perp}^{2} f_{a_{0} a_{1} c}\left(n^{+}\right)^{\nu_{1}} \frac{1}{k_{0}^{+}}\left(n^{+}\right)^{\nu_{0}}, \quad k_{0}^{+}+k_{1}^{+}=0,
\end{aligned}
$$




$$
\begin{aligned}
& \Delta_{a_{0} a_{1} a_{2} c}^{v_{0} v_{1} v_{2}}\left(k_{0}^{+}, k_{1}^{+}, k_{2}^{+}\right)=-\boldsymbol{q}_{\perp}^{2}\left(n^{+}\right)^{\nu_{0}}\left(n^{+}\right)^{v_{1}}\left(n^{+}\right)^{v_{2}}\left(\frac{f_{a_{2} a_{0} a} f_{a_{1} a c}}{k_{1}^{+} k_{2}^{+}}+\frac{f_{a_{2} a_{1} a} f_{a_{0} a c}}{k_{0}^{+} k_{2}^{+}}\right), \\
& k_{0}^{+}+k_{1}^{+}+k_{2}^{+}=0 .
\end{aligned}
$$

The last vertex is Bose-symmetric due to the Jacobi identity.

It is helpful to introduce the operators $G_{a_{0} a_{1} \ldots a_{r}}\left(k_{0}^{+}, k_{1}^{+}, \ldots, k_{r}^{+}\right)$using the definition

$$
\begin{aligned}
& \Delta_{a_{0} a_{1} \ldots a_{r} c}^{v_{0} v_{1} \ldots v_{r}+}\left(k_{0}^{+}, k_{1}^{+}, \ldots, k_{r}^{+}\right) \\
& \quad=-2 q^{2}\left(n^{+}\right)^{v_{0}}\left(n^{+}\right)^{v_{1}} \ldots\left(n^{+}\right)^{v_{r}} \operatorname{Tr}\left[T^{c} G_{a_{0} a_{1} \ldots a_{r}}\left(k_{0}^{+}, k_{1}^{+}, \ldots, k_{r}^{+}\right)\right], \quad r \geqslant 2 .
\end{aligned}
$$

They can be constructed from the above effective action for arbitrary $r$ in the generalized eikonal form

$$
\begin{aligned}
& G_{a_{0} a_{1} \ldots a_{r}}\left(k_{0}^{+}, k_{1}^{+}, \ldots, k_{r}^{+}\right) \\
& \quad=(-1)^{r} \sum_{\left\{i_{0}, i_{1}, \ldots, i_{r}\right\}} \frac{T^{a_{i}} T^{a_{i_{1}}} T^{a_{i_{2}}} \cdots T^{a_{i_{r}}}}{k_{i_{0}}^{+}\left(k_{i_{0}}^{+}+k_{i_{1}}^{+}\right)\left(k_{i_{0}}^{+}+k_{i_{1}}^{+}+k_{i_{2}}^{+}\right) \cdots\left(k_{i_{0}}^{+}+k_{i_{1}}^{+}+\cdots+k_{i_{r-1}}^{+}\right)},
\end{aligned}
$$

where the sum is performed over all permutations of numbers $0,1, \ldots, r$. Note, that this expression is explicitly Bose symmetric. Due to the gauge invariance of the effective action, the vertices $G$ 's satisfy the Ward identity in the form

$$
\begin{aligned}
k_{r}^{+} & G_{a_{0} a_{1} \ldots a_{r}}\left(k_{0}^{+}, k_{1}^{+}, \ldots, k_{r}^{+}\right) \\
& =-i \sum_{i=0}^{r-1} f_{a_{r} a_{i} a} G_{a_{0} a_{1} \ldots a_{i-1} a a_{i+1} \ldots a_{r-1}}\left(k_{0}^{+}, k_{1}^{+}, \ldots, k_{i-1}^{+}, k_{i}^{+}+k_{r}^{+}, k_{i+1}^{+}, \ldots, k_{r-1}^{+}\right),
\end{aligned}
$$

where we used the following relations for $i_{t}=r$

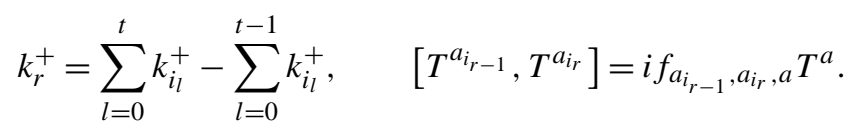

The above identity does not depend on the color representation of the generators $T^{a}$ in expression (19) for $G$. It means, that we can use for the Wilson $\mathcal{P}$-exponentials in Eq. (16) an arbitrary representation providing that the matrices $A_{ \pm}$are taken in the same representation and the corresponding trace normalization is chosen.

Thus, we obtain the following recurrent relations for the effective vertices $\Delta_{a_{0} a_{1} \ldots a_{r} c}^{v_{0} v_{1} \ldots v_{r}+}\left(k_{0}^{+}, k_{1}^{+}, \ldots, k_{r}^{+}\right)[8]:$

$$
\begin{aligned}
\Delta_{a_{0} a_{1} \ldots a_{r}}^{v_{0} v_{1} \ldots v_{r}} & \left(k_{0}^{+}, k_{1}^{+}, \ldots, k_{r}^{+}\right) \\
= & \frac{-i}{k_{r}^{+}}\left(n^{+}\right)^{v_{r}} \\
& \times \sum_{i=0}^{r-1} f_{a_{r} a_{i} a} \Delta_{a_{0} a_{1} \ldots a_{i-1} a a_{i+1} \ldots a_{r-1}}^{\nu_{0} \nu_{1} \ldots v_{r-1}+}\left(k_{0}^{+}, k_{1}^{+}, \ldots, k_{i-1}^{+}, k_{i}^{+}+k_{r}^{+}, k_{i+1}^{+}, \ldots, k_{r-1}^{+}\right) .
\end{aligned}
$$




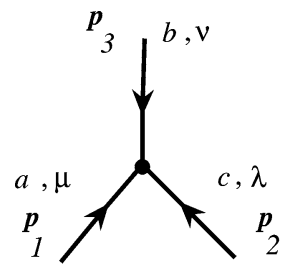

(a)

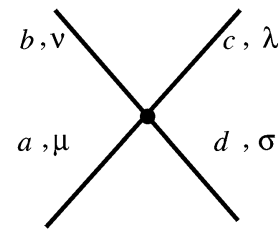

(b)

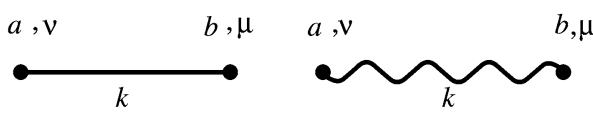

(c)

(d)

Fig. 2. Standard QCD Feynman rules: vertices (a)-(c), and the reggeized gluon propagator (d).

In the next section, we construct the Feynman rules for the reggeon vertices in an agreement with the above recurrence relations.

\section{Vertices}

\subsection{Standard QCD Feynman rules}

The Yang-Mills interaction part of the effective action yields the standard QCD Feynman rules (see Fig. 2(a)-(c)):

$$
\begin{gathered}
3 g \text {-vertex: } \quad g f_{a b c}\left[\left(p_{1}-p_{2}\right)_{\lambda} g_{\mu \nu}+\left(p_{2}-p_{3}\right)_{\mu} g_{\nu \lambda}+\left(p_{3}-p_{1}\right)_{\nu} g_{\lambda \mu}\right] \\
\equiv g f_{a b c} \gamma_{\mu \nu \lambda}\left(p_{1}, p_{2}, p_{3}\right),
\end{gathered}
$$

having the property

$$
\begin{aligned}
& \begin{aligned}
\gamma_{\mu \nu \lambda}\left(p_{1}, p_{2}, p_{3}\right)= & \gamma_{\lambda \mu \nu}\left(p_{3}, p_{1}, p_{2}\right)=-\gamma_{\nu \mu \lambda}\left(p_{2}, p_{1}, p_{3}\right)=\cdots, \\
4 g \text {-vertex: } \quad i g^{2}[ & f_{a b l} f_{c d l}\left(g^{\mu \sigma} g^{\nu \lambda}-g^{\mu \lambda} g^{\nu \sigma}\right)+f_{a c l} f_{d b l}\left(g^{\mu \nu} g^{\lambda \sigma}-g^{\mu \sigma} g^{\lambda \nu}\right) \\
& \left.+f_{a d l} f_{b c l}\left(g^{\mu \lambda} g^{\sigma \nu}-g^{\mu \nu} g^{\sigma \lambda}\right)\right] \\
\equiv & i g^{2} \gamma_{a b c d}^{\mu \nu \lambda \sigma}, \quad \gamma_{a b c d}^{\mu \nu \lambda \sigma}=\gamma_{b a c d}^{\nu \mu \lambda \sigma}=\cdots,
\end{aligned} \\
& \text { gluon propagator: } \quad-i \delta^{a b} \frac{g_{\mu \nu}}{k^{2}}, \\
& \text { quark-gluon vertex: } \quad i g \bar{u}\left(p_{1}\right) \gamma^{\mu} T^{a} u\left(p_{2}\right), \\
& \text { quark propagator: } i \frac{\hat{p}+m}{p^{2}-m^{2}} .
\end{aligned}
$$

In addition, we specify the reggeized gluon propagator, Fig. 2(d):

$$
-\frac{i}{2 k^{2}} \delta^{a b}\left[\left(n^{+}\right)^{\mu}\left(n^{-}\right)^{v}+\left(n^{+}\right)^{v}\left(n^{-}\right)^{\mu}\right] .
$$

\subsection{Effective PPR vertices}

Knowledge of the induced and ordinary 3-vertices allows one to build the effective 3vertices which obey the Bose- and gauge-symmetries. We use here the conservation laws in the form of Eqs. (8), (9). 
We distinguish four types of the $P P R$ vertices. First group consists of the margin type vertices: $q_{2}=-P_{A}+P_{A^{\prime}}, q_{1}=P_{B^{\prime}}-P_{B}$.

- "Left" margin type (see Fig. 4(a)):

$$
\begin{aligned}
\gamma_{\| a b c}^{v v^{\prime}+}\left(P_{A}, a ; P_{A^{\prime}}, b ; q_{2}, c\right) & =g f_{a b c} \Gamma^{v v^{\prime}+}\left(P_{A}, q_{2}\right), \\
\Gamma^{v v^{\prime}+}\left(P_{A}, q_{2}\right)= & 2 P_{A}^{+} g^{v v^{\prime}}+\left(n^{+}\right)^{v}\left(-2 P_{A}+P_{A^{\prime}}\right)^{v^{\prime}} \\
& +\left(n^{+}\right)^{v^{\prime}}\left(-2 P_{A^{\prime}}+P_{A}\right)^{v}-\frac{q_{2}^{2}}{P_{A}^{+}}\left(n^{+}\right)^{v}\left(n^{+}\right)^{v^{\prime}} .
\end{aligned}
$$

One can check that the condition of gauge invariance is explicitly satisfied:

$$
\begin{aligned}
& \Gamma^{v v^{\prime}+}\left(P_{A}, q_{2}\right) \cdot\left(P_{A^{\prime}}\right)_{v^{\prime}}=P_{A}^{v} P_{A}^{+}-P_{A}^{2}\left(n^{+}\right)^{v}, \\
& \Gamma^{v v^{\prime}+}\left(P_{A}, q_{2}\right) \cdot\left(P_{A}\right)_{v}=P_{A^{\prime}}^{v^{\prime}} P_{A}^{+}-P_{A^{\prime}}^{2}\left(n^{+}\right)^{v^{\prime}} .
\end{aligned}
$$

For on-mass-shell particle $A: P_{A}^{2}=0$ with the gauge condition $\left(e\left(P_{A}\right) \cdot P_{A}\right)=0$ we have

$$
\Gamma^{v v^{\prime}+}\left(P_{A}, q_{2}\right) \cdot\left(P_{A^{\prime}}\right)_{v^{\prime}} \cdot e_{v}\left(P_{A}\right)=0 .
$$

- "Right" margin type (see Fig. 4(b)):

$$
\begin{aligned}
& \gamma_{\|}^{v v^{\prime}-}\left(P_{B}, a ; P_{B^{\prime}}, b ; q_{1}, c\right)=-g f_{b a c} \Gamma^{v v^{\prime}-}\left(q_{1}, P_{B}\right), \\
& \Gamma^{v v^{\prime}-}\left(q_{1}, P_{B}\right)= 2 P_{B}^{-} g^{v v^{\prime}}-\left(n^{-}\right)^{v^{\prime}}\left(2 P_{B^{\prime}}-P_{B}\right)^{v}-\left(n^{-}\right)^{v}\left(2 P_{B}-P_{B^{\prime}}\right)^{v^{\prime}} \\
&-\frac{q_{1}^{2}}{P_{B}^{-}}\left(n^{-}\right)^{v}\left(n^{-}\right)^{v^{\prime}} .
\end{aligned}
$$

In this case, the gauge-invariance tests read

$$
\begin{aligned}
& \Gamma^{\nu v^{\prime}-}\left(q_{1}, P_{B}\right) \cdot\left(P_{B^{\prime}}\right)_{v^{\prime}}=P_{B}^{v} P_{B}^{-}-P_{B}^{2}\left(n^{-}\right)^{v}, \\
& \Gamma^{v v^{\prime}-}\left(q_{1}, P_{B}\right) \cdot\left(P_{B}\right)_{v}=P_{B^{\prime}}^{v^{\prime}} P_{B}^{-}-P_{B^{\prime}}^{2}\left(n^{-}\right)^{v^{\prime}} .
\end{aligned}
$$

The second group includes the effective vertices of the central type where $k=q_{1}+q_{2}$, $q_{1,2}^{2} \neq 0$ :

- "Left" central type (see Fig. 4(c)):

$$
\begin{aligned}
& \gamma_{\perp a b c}^{v v^{\prime}+}\left(q_{1}, a ; k, b ; q_{2}, c\right)=g f_{a b c} \Gamma^{\nu v^{\prime}+}\left(q_{1}, q_{2}\right), \\
& \Gamma^{\nu v^{\prime}+}\left(q_{1}, q_{2}\right)= 2 q_{1}^{+} g^{v v^{\prime}}-\left(n^{+}\right)^{v}\left(q_{1}-q_{2}\right)^{v^{\prime}}-\left(n^{+}\right)^{v^{\prime}}\left(q_{1}+2 q_{2}\right)^{v} \\
&-\frac{q_{2}^{2}}{q_{1}^{+}}\left(n^{+}\right)^{v}\left(n^{+}\right)^{v^{\prime}} .
\end{aligned}
$$

The corresponding Ward identities read:

$$
\begin{aligned}
& \Gamma^{v v^{\prime}+}\left(q_{1}, q_{2}\right) \cdot k_{v^{\prime}}=q_{1}^{+} q_{1}^{v}-q_{1}^{2}\left(n^{+}\right)^{v}, \\
& \Gamma^{v v^{\prime}+}\left(q_{1}, q_{2}\right) \cdot\left(q_{1}\right)_{v}=q_{1}^{+} k^{v^{\prime}}-k^{2}\left(n^{+}\right)^{v^{\prime}} .
\end{aligned}
$$


- "Right" central type (see Fig. 4(d)):

$$
\begin{aligned}
& \gamma_{\perp a b c}^{v v^{\prime}-}\left(q_{1}, a ; k, b ; q_{2}, c\right)=-g f_{a b c} \Gamma^{\nu v^{\prime}-}\left(q_{1}, q_{2}\right), \\
& \Gamma^{\nu v^{\prime}-}\left(q_{1}, q_{2}\right)= 2 q_{2}^{-} g^{v v^{\prime}}+\left(n^{-}\right)^{v}\left(q_{1}-q_{2}\right)^{v^{\prime}}+\left(n^{-}\right)^{v^{\prime}}\left(-q_{2}-2 q_{1}\right)^{v} \\
&-\frac{q_{1}^{2}}{q_{2}^{-}}\left(n^{-}\right)^{v}\left(n^{-}\right)^{v^{\prime}},
\end{aligned}
$$

for which one has

$$
\begin{aligned}
& \Gamma^{v v^{\prime}-}\left(q_{1}, q_{2}\right) \cdot k_{v^{\prime}}=q_{2}^{-} q_{2}^{v}-\left(n^{-}\right)^{v} q_{2}^{2}, \\
& \Gamma^{v v^{\prime}-}\left(q_{1}, q_{2}\right) \cdot\left(q_{2}\right)_{v}=q_{2}^{-} k^{v^{\prime}}-k^{2}\left(n^{-}\right)^{v^{\prime}} .
\end{aligned}
$$

\subsection{Effective PRR vertex}

Production of a single gluon with momentum $k_{\mu}=\left(q_{1}+q_{2}\right)_{\mu}$ and color index $b$ in the "two reggeons collision" in color-momentum states, respectively, $\left(q_{1}, a ; k, b ; q_{2} c\right)$, is described by the $P R R$ vertex (see Fig. 4(e))

$$
\begin{aligned}
& \Gamma^{-\mu+}\left(q_{1}, a ; k, b ; q_{2}, c\right)=g f_{a b c} C^{\mu}\left(q_{1}, q_{2}\right), \\
& f_{a b c} C^{\mu}\left(q_{1}, q_{2}\right)= f_{a b c} \gamma^{\nu \mu \eta}\left(q_{1},-k, q_{2}\right)\left(n^{-}\right)_{\nu}\left(n^{+}\right)_{\eta}+\Delta_{c b a}^{-\mu \eta}\left(q_{1}, k, q_{2}\right)\left(n^{+}\right)_{\eta} \\
&-\Delta_{a b c}^{\eta \mu+}\left(q_{1}, k, q_{2}\right)\left(n^{-}\right)_{\eta} .
\end{aligned}
$$

As a result we have

$$
C^{\mu}\left(q_{1}, q_{2}\right)=2\left[\left(n^{-}\right)^{\mu}\left(q_{1}^{+}+\frac{q_{1}^{2}}{q_{2}^{-}}\right)-\left(n^{+}\right)^{\mu}\left(q_{2}^{-}+\frac{q_{2}^{2}}{q_{1}^{+}}\right)+\left(q_{2}-q_{1}\right)^{\mu}\right] .
$$

The 4-vector $C^{\mu}$ obeys the gauge condition $k_{\mu} \cdot C^{\mu}\left(q_{1}, q_{2}\right)=0$.

\subsection{Effective RRP P vertex}

We consider first the case when pair of gluons in color-momenta states $\left(p_{1}, v_{1}, a_{1} ; p_{2}\right.$, $\left.v_{2}, a_{2}\right)$ are created in collision of two reggeons with color-momenta states $\left(q_{1}, c ; q_{2}, d\right)$ with the momentum conservation relation $q_{1}+q_{2}=p_{1}+p_{2}$, Fig. 5 . It can be build in terms of the effective 3-vertices given above (see Fig. 4) It has the form

$$
\begin{aligned}
& \frac{1}{i g^{2}} \Gamma_{c a_{1} a_{2} d}^{-v_{1} v_{2}+}\left(q_{1} ; p_{1}, p_{2} ; q_{2}\right) \\
&=\frac{T_{1}}{p_{12}^{2}} C^{\eta}\left(q_{1}, q_{2}\right) \gamma^{\nu_{1} v_{2} \eta}\left(-p_{1},-p_{2}, p_{12}\right) \\
& \quad+\frac{T_{3}}{\left(p_{2}-q_{2}\right)^{2}} \Gamma^{\eta v_{1}-}\left(q_{1}, p_{1}-q_{1}\right) \Gamma^{\eta v_{2}+}\left(p_{2}-q_{2}, q_{2}\right) \\
&-\frac{T_{2}}{\left(p_{1}-q_{2}\right)^{2}} \Gamma^{\eta v_{2}-}\left(q_{1}, p_{2}-q_{1}\right) \Gamma^{\eta v_{1}+}\left(p_{1}-q_{2}, q_{2}\right) \\
&-T_{1}\left[\left(n^{-}\right)^{v_{1}}\left(n^{+}\right)^{\nu_{2}}-\left(n^{-}\right)^{\nu_{2}}\left(n^{+}\right)^{v_{1}}\right]-T_{2}\left[2 g^{v_{1} v_{2}}-\left(n^{-}\right)^{v_{1}}\left(n^{+}\right)^{\nu_{2}}\right]
\end{aligned}
$$




$$
\begin{aligned}
& -T_{3}\left[\left(n^{-}\right)^{\nu_{2}}\left(n^{+}\right)^{\nu_{1}}-2 g^{\nu_{1} \nu_{2}}\right] \\
& +\Delta_{c a_{1} a_{2} d}^{\rho v_{1} \nu_{2}+}\left(q_{1}, p_{1}, p_{2}, q_{2}\right)\left(n^{-}\right)_{\rho}+\Delta_{c a_{1} a_{2} d}^{-v_{1} \nu_{2} \eta}\left(q_{1}, p_{1}, p_{2}, q_{2}\right)\left(n^{+}\right)_{\eta},
\end{aligned}
$$

where

$$
T_{1}=f_{a_{1} a_{2} r} f_{c d r}, \quad T_{2}=f_{a_{2} c r} f_{a_{1} d r}, \quad T_{3}=f_{c a_{1} r} f_{a_{2} d r}, \quad T_{1}+T_{2}+T_{3}=0,
$$

and the induced vertices, Fig. 3(e):

$$
\begin{aligned}
& \Delta_{c a_{1} a_{2} d}^{\rho v_{1} v_{2}+}\left(q_{1}, p_{1}, p_{2}, q_{2}\right)\left(n^{-}\right)_{\rho}=-2 q_{2}^{2}\left(n^{+}\right)^{v_{1}}\left(n^{+}\right)^{\nu_{2}}\left(\frac{T_{3}}{p_{2}^{+} q_{1}^{+}}-\frac{T_{2}}{p_{1}^{+} q_{1}^{+}}\right), \\
& \Delta_{c a_{1} a_{2} d}^{-v_{1} v_{2} \eta}\left(q_{1}, p_{1}, p_{2}, q_{2}\right)\left(n^{+}\right)_{\eta}=-2 q_{1}^{2}\left(n^{-}\right)^{v_{1}}\left(n^{-}\right)^{v_{2}}\left(\frac{T_{3}}{p_{1}^{-} q_{2}^{-}}-\frac{T_{2}}{p_{2}^{-} q_{2}^{-}}\right) .
\end{aligned}
$$

One can verify the fulfillment of the gauge- and Bose-symmetries requirements:

$$
\begin{aligned}
& \Gamma_{c a_{1} a_{2} d}^{-v_{1} v_{2}+}\left(q_{1} ; p_{1}, p_{2} ; q_{2}\right) p_{1 v_{1}}=0, \\
& \Gamma_{c a_{1} a_{2} d}^{-v_{1} v_{2}+}\left(q_{1} ; p_{1}, p_{2} ; q_{2}\right)=\Gamma_{c a_{2} a_{1} d}^{-v_{2} v_{1}+}\left(q_{1} ; p_{2}, p_{1} ; q_{2}\right) .
\end{aligned}
$$
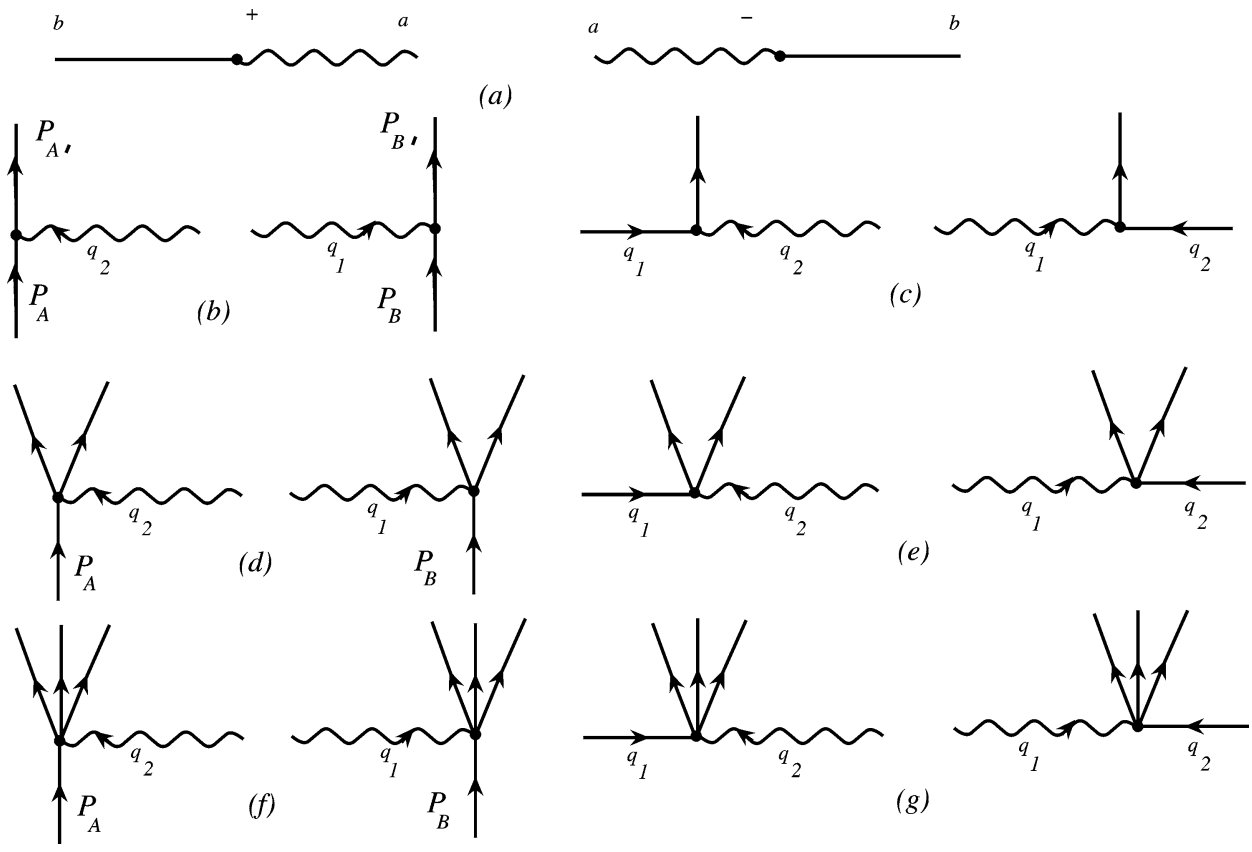

(g)

Fig. 3. List of the induced vertices. 


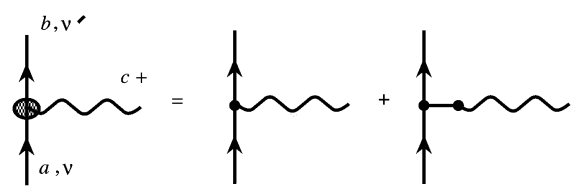

(a)

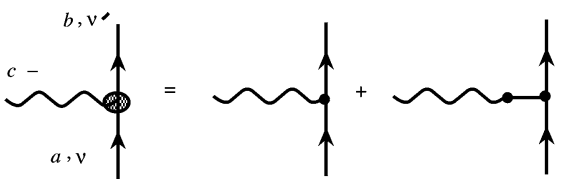

(b)

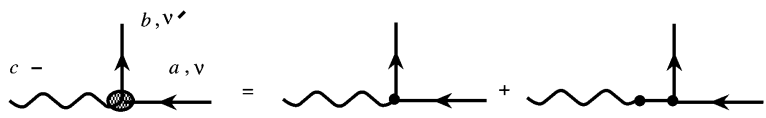

(c)

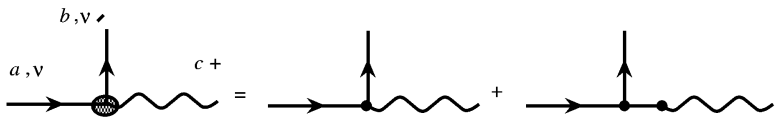

(d)

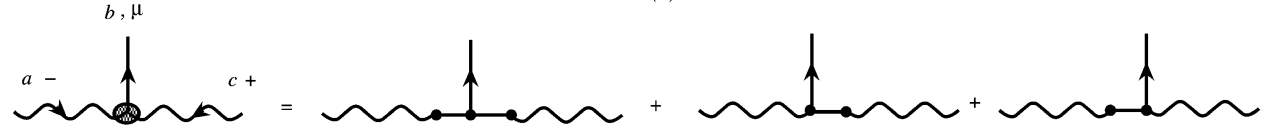

(e)

Fig. 4. $P P R$ (a)-(d) and $R R P$ (e) effective vertices.
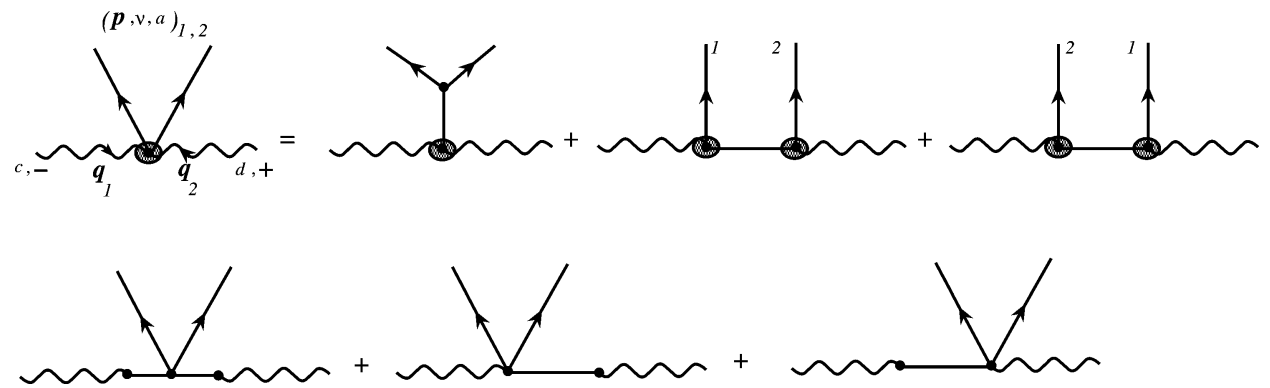

Fig. 5. Central $P P R R$ effective vertex.

\subsection{Effective PPPR vertices}

Let us consider now the margin $P P P R$ vertices. The effective $P P P R$ vertex can be constructed in terms of combination of previously calculated effective vertices, and ordinary 3-vertices (see Figs. 6, 7).

For the vertex describing the $A$-particle fragmentation region to two gluons and a reggeon $P_{A}+R\left(q_{2}\right) \rightarrow g_{1}\left(p_{1}\right)+g_{2}\left(p_{2}\right)$ (see Fig. 6), one has

$$
\begin{aligned}
& \frac{1}{i g^{2}} \Gamma_{c a_{1} a_{2} d}^{\nu_{0} \nu_{1} \nu_{2}+}\left(P_{A}, p_{1}, p_{2}, q_{2}\right) \\
& \quad=\frac{T_{1}}{p_{12}^{2}} \gamma^{\sigma \nu_{1} \nu_{2}}\left(p_{12},-p_{1},-p_{2}\right) \Gamma^{\nu_{0} \sigma+}\left(P_{A}, q_{2}\right)
\end{aligned}
$$




$$
\begin{aligned}
& +\frac{T_{3}}{\left(p_{2}-q_{2}\right)^{2}} \gamma^{\nu_{0} v_{1} \sigma}\left(P_{A},-p_{1}, p_{1}-P_{A}\right) \Gamma^{\sigma \nu_{2}+}\left(p_{2}-q_{2}, q_{2}\right) \\
& +\frac{T_{2}}{\left(p_{1}-q_{2}\right)^{2}} \gamma^{\nu_{0} \nu_{2} \sigma}\left(P_{A},-p_{2}, p_{2}-P_{A}\right) \Gamma^{\sigma \nu_{1}+}\left(p_{1}-q_{2}, q_{2}\right) \\
& +T_{3}\left[g^{\nu_{1} \nu_{2}}\left(n^{+}\right)^{\nu_{0}}-\left(n^{+}\right)^{\nu_{1}} g^{\nu_{0} \nu_{2}}\right]+T_{2}\left[g^{\nu_{0} \nu_{1}}\left(n^{+}\right)^{\nu_{2}}-g^{\nu_{1} \nu_{2}}\left(n^{+}\right)^{\nu_{0}}\right] \\
& +T_{1}\left[\left(n^{+}\right)^{\nu_{1}} g^{\nu_{0} \nu_{2}}-\left(n^{+}\right)^{\nu_{2}} g^{\nu_{0} \nu_{1}}\right]+\Delta_{c a_{1} a_{2} d}^{\nu_{0} v_{1} \nu_{2}+}\left(p_{A}, p_{1}, p_{2}, q_{2}\right),
\end{aligned}
$$

with the same definition for $T_{i}$ 's as for the case $R R P P$ effective vertex, Eq. (44), and the conservation law $P_{A}+q_{2}=p_{1}+p_{2}$. When checking the gauge invariance (convoluting with $\left.\left(p_{1}\right)_{v_{1}}\right)$ we use the expression for the induced vertex (see Fig. 3(d) left)

$$
\Delta_{c a_{1} a_{2} d}^{v_{0} v_{1} v_{2}+}\left(p_{A}, p_{1}, p_{2}, q_{2}\right)=\frac{q_{2}^{2}}{P_{A}^{+}}\left[\frac{T_{2}}{p_{1}^{+}}-\frac{T_{3}}{p_{2}^{+}}\right]\left(n^{+}\right)^{v_{0}}\left(n^{+}\right)^{v_{1}}\left(n^{+}\right)^{\nu_{2}} .
$$

One can verify the fulfillment of the gauge condition:

$$
\Gamma_{c a_{1} a_{2} d}^{\nu_{0} v_{1} \nu_{2}+}\left(P_{A}, p_{1}, p_{2}, q_{2}\right) \cdot\left(p_{1}\right)_{v_{1}}=0
$$

as well as the Bose-symmetry condition:

$$
\Gamma_{c a_{1} a_{2} d}^{\nu_{0} \nu_{1} \nu_{2}+}\left(P_{A}, p_{1}, p_{2}, q_{2}\right)=\Gamma_{c a_{2} a_{1} d}^{\nu_{0} \nu_{2} v_{1}+}\left(P_{A}, p_{2}, p_{1}, q_{2}\right)=\cdots .
$$

Similarly, we find for the "right" effective margin vertex, Fig. 7:

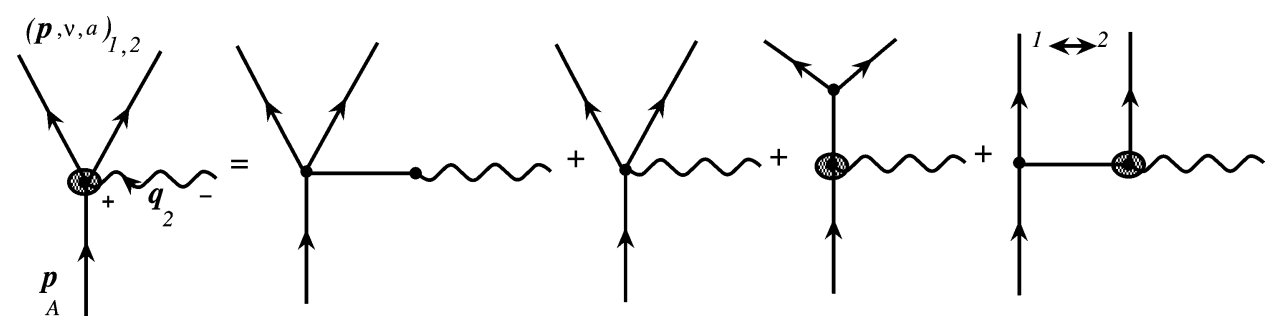

Fig. 6. Margin P P P R effective vertex: "left" type.

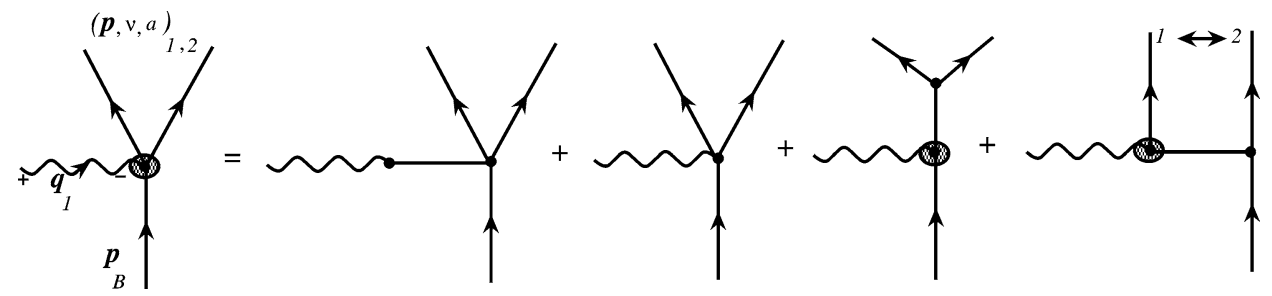

Fig. 7. Margin $P P P R$ effective vertex: "right" type. 


$$
\begin{aligned}
& \frac{1}{i g^{2}} \Gamma_{c a_{1} a_{2} d}^{-v_{1} v_{2} v_{0}}\left(q_{1}, p_{1}, p_{2}, P_{B}\right) \\
& =-\frac{T_{1}}{p_{12}^{2}} \gamma^{\sigma v_{1} \nu_{2}}\left(p_{12},-p_{1},-p_{2}\right) \Gamma^{\nu_{0} \sigma-}\left(q_{1}, p_{12}-q_{1}\right) \\
& +\frac{T_{3}}{\left(p_{1}-q_{1}\right)^{2}} \gamma^{\sigma v_{2} v_{0}}\left(p_{2}-P_{B},-p_{2}, P_{B}\right) \Gamma^{\sigma v_{1}-}\left(q_{1}, p_{1}-q_{1}\right) \\
& -\frac{T_{2}}{\left(p_{2}-q_{1}\right)^{2}} \gamma^{\sigma v_{1} \nu_{0}}\left(p_{1}-P_{B},-p_{1}, P_{B}\right) \Gamma^{\sigma \nu_{2}-}\left(q_{1}, p_{2}-q_{1}\right) \\
& +T_{1}\left[\left(n^{-}\right)^{\nu_{2}} g^{\nu_{0} \nu_{1}}-\left(n^{-}\right)^{\nu_{1}} g^{\nu_{0} \nu_{2}}\right]+T_{3}\left[g^{\nu_{1} \nu_{2}}\left(n^{-}\right)^{\nu_{0}}-\left(n^{-}\right)^{\nu_{2}} g^{\nu_{0} \nu_{1}}\right] \\
& +T_{2}\left[\left(n^{-}\right)^{\nu_{1}} g^{\nu_{0} \nu_{2}}-\left(n^{-}\right)^{\nu_{0}} g^{\nu_{1} \nu_{2}}\right]+\Delta_{c a_{1} a_{2} d}^{-\nu_{1} \nu_{2} \nu_{0}}\left(q_{1}, p_{1}, p_{2}, P_{B}\right),
\end{aligned}
$$

with the same notations for $T_{i}$ 's as for "left" margin vertex and the $R R P P$ vertex, Eq. (44), conservation law $q_{1}+P_{B}=p_{1}+p_{2}$, and the induced vertex, Fig. 3(d) right:

$$
\Delta_{c a_{1} a_{2} d}^{-v_{1} v_{2} v_{0}}\left(q_{1}, p_{1}, p_{2}, P_{B}\right)=\frac{q_{1}^{2}}{P_{B}^{-}}\left[\frac{T_{2}}{p_{2}^{-}}-\frac{T_{3}}{p_{1}^{-}}\right]\left(n^{-}\right)^{v_{0}}\left(n^{-}\right)^{v_{1}}\left(n^{-}\right)^{\nu_{2}} .
$$

Again, the gauge condition is fulfilled:

$$
\Gamma_{d a_{1} a_{2} c}^{-v_{1} v_{2} v_{0}}\left(q_{1}, p_{1}, p_{2}, P_{B}\right) \cdot\left(p_{1}\right)_{v_{1}}=0,
$$

as well as the Bose-symmetry.

\subsection{Effective RRP P P, and RP P P P vertices}

- Consider first the $R R P P P$ vertex $V^{+v_{1} v_{2} v_{3}-}\left(q_{1}, p_{1}, p_{2}, p_{3}, q_{2}\right)$. It can be constructed by means of combinations of the already known effective vertices given above, Eqs. (23), (25), (26), (37), (39). The result is graphically presented at the Fig. 8. Each of these diagrams presents, indeed, a set of diagrams related to each other by any possible permutations of the produced particles. Using the rules given in Appendix A, one can write down the analytical expression summing up all these contributions:

$$
\begin{aligned}
\frac{1}{g^{3}} \Gamma_{d 123 e}^{-v_{1} v_{2} v_{3}+}\left(q_{1}, p_{1}, p_{2}, p_{3}, q_{2}\right) \\
=\frac{f_{e d r}}{k^{2}} C_{\sigma}\left(q_{1}, q_{2}\right) \cdot A_{a_{1} a_{2} a_{3} r}^{\nu_{1} \nu_{2} \nu_{3} \sigma} \\
\quad+\left[f_{m d r} f_{r 13} f_{e m 2} \frac{1}{p_{13}^{2}\left(p_{2}-q_{2}\right)^{2}} \Gamma^{\sigma \eta-}\left(q_{1}, p_{13}-q_{1}\right)\right. \\
\left.\quad \times \Gamma^{\sigma v_{2}+}\left(p_{2}-q_{2}, q_{2}\right) \gamma^{\nu_{1} v_{3} \eta}\left(-p_{1},-p_{3}, p_{13}\right)+2 \text { perm. }\right] \\
+ \\
\quad\left[f_{m d 3} f_{r 12} f_{e m r} \frac{1}{p_{12}^{2}\left(p_{3}-q_{1}\right)^{2}} \Gamma^{\sigma \nu_{3}-}\left(q_{1}, p_{3}-q_{1}\right)\right. \\
\left.\quad \times \Gamma^{\sigma \eta+}\left(p_{12}-q_{2}, q_{2}\right) \gamma^{\nu_{1} v_{2} \eta}\left(-p_{1},-p_{2}, p_{12}\right)+2 \text { perm. }\right]
\end{aligned}
$$




$$
\begin{aligned}
& +\left[f_{m d 1} f_{r m 2} f_{e r 3} \frac{1}{\left(q_{1}-p_{1}\right)^{2}\left(p_{3}-q_{2}\right)^{2}} \Gamma^{\sigma \nu_{1}-}\left(q_{1}, p_{1}-q_{1}\right)\right. \\
& \left.\times \Gamma^{\eta \nu_{3}+}\left(p_{3}-q_{2}, q_{2}\right) \gamma^{\sigma \nu_{2} \eta}\left(q_{1}-p_{1},-p_{2}, q_{2}-p_{3}\right)+5 \text { perm. }\right] \\
& +\left[f_{e r 3} \frac{1}{\left(p_{3}-q_{2}\right)^{2}} \Gamma^{\sigma \nu_{3}+}\left(p_{3}-q_{2}, q_{2}\right)(\Delta+\gamma)_{d 12 r}^{-v_{1} \nu_{2} \sigma}\left(q_{1}, p_{1}, p_{2}, q_{2}-p_{3}\right)\right. \\
& +2 \text { perm. }] \\
& -\left[f_{r d 3} \frac{1}{\left(p_{3}-q_{1}\right)^{2}} \Gamma^{\sigma \nu_{3}-}\left(q_{1}, p_{3}-q_{1}\right)(\Delta+\gamma)_{r 12 e}^{\sigma \nu_{1} \nu_{2}+}\left(q_{1}-p_{3}, p_{1}, p_{2}, q_{2}\right)\right. \\
& +2 \text { perm. }] \\
& +\left[f_{r 23} \frac{1}{p_{23}^{2}} \gamma^{\nu_{2} \nu_{3} \sigma}\left(-p_{2}-p_{3}, p_{23}\right)\right. \\
& \times\left(\Delta_{d r 1 e}^{-\sigma \nu_{1} \eta} \cdot\left(n^{+}\right)_{\eta}+\gamma_{d r 1 e}^{-\sigma \nu_{1}+}+\Delta_{d r 1 e}^{\rho \sigma \nu_{1}+} \cdot\left(n^{-}\right)_{\rho}\right)\left(q_{1}, p_{23}, p_{1}, q_{2}\right) \\
& +2 \text { perm. } \\
& +\left(\Delta_{d 123 e}^{-v_{1} v_{2} v_{3} \rho} \cdot\left(n^{+}\right)_{\rho}+\Delta_{d 123 e}^{\rho v_{1} v_{2} v_{3}+} \cdot\left(n^{-}\right)_{\rho}\right)\left(q_{1}, p_{1}, p_{2}, p_{3}, q_{2}\right),
\end{aligned}
$$

where we use the shorthand notations

$$
f_{123} \equiv f_{a_{1} a_{2} a_{3},} \quad p_{12}=p_{1}+p_{2}, \quad k=p_{1}+p_{2}+p_{3} .
$$
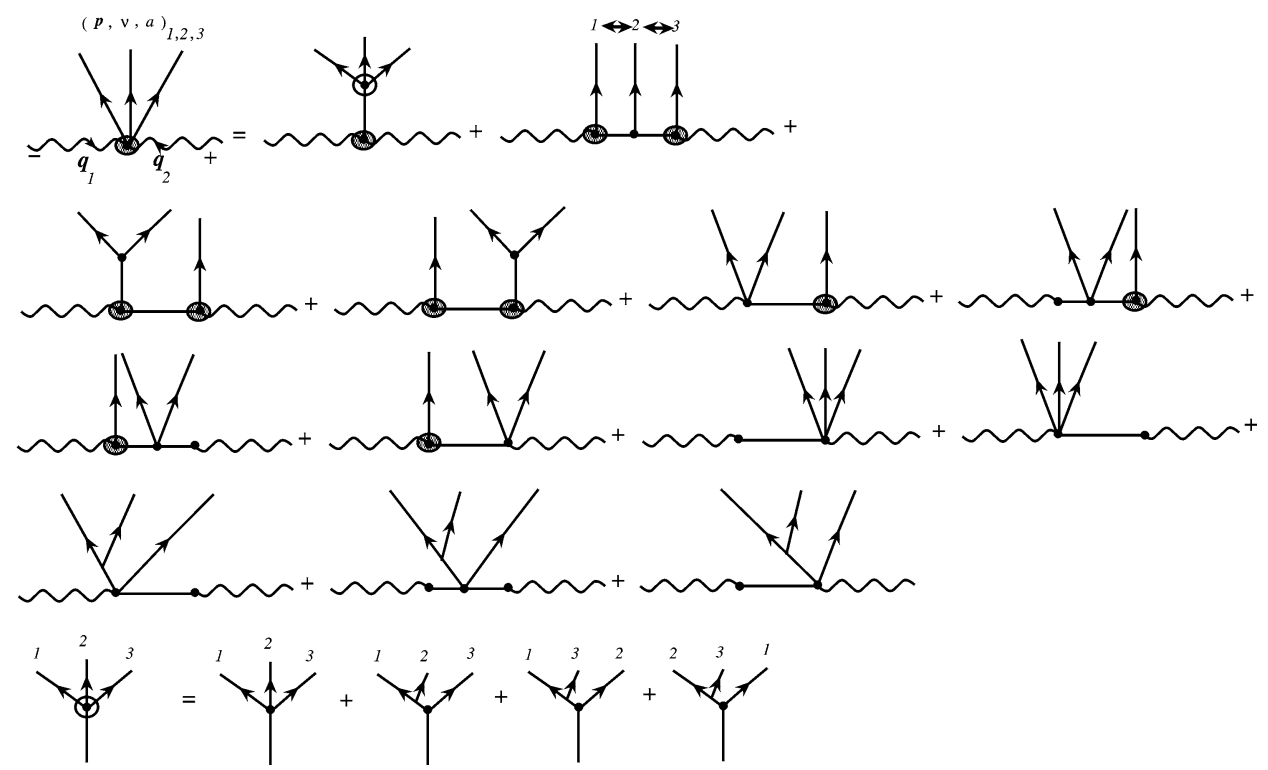

Fig. 8. Central $R R P P P$ effective vertex. 

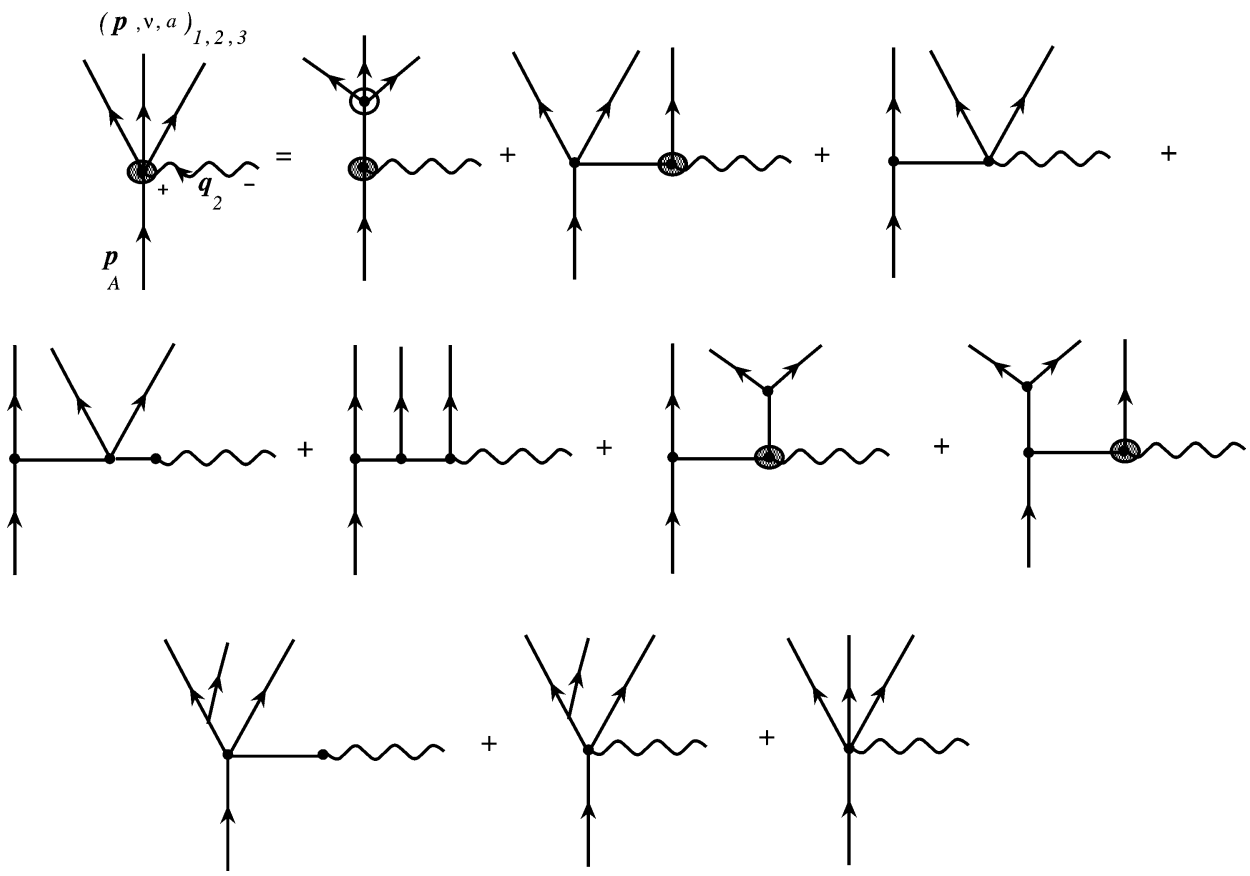

Fig. 9. Margin P P P PR effective vertex: "left" type.

For the induced terms of the rank-2 entering $R R P P P$ we have (see Fig. 3(g)):

$$
\begin{aligned}
& \Delta_{d 12 r}^{-v_{1} \nu_{2} \sigma}\left(q_{1}, p_{1}, p_{2}, q_{2}-p_{3}\right) \\
& =-\frac{q_{1}^{2}}{p_{1}^{-}}\left(n^{-}\right)^{\nu_{1}}\left(n^{-}\right)^{\nu_{2}}\left(n^{-}\right)^{\sigma}\left[\frac{f_{1 r m} f_{2 d m}}{p_{2}^{-}}+\frac{f_{r d m} f_{12 m}}{p_{12}^{-}}\right], \\
& \Delta_{d r 1 e}^{-\sigma \nu_{1} \eta}\left(q_{1}, p_{23}, p_{1}, q_{2}\right) \\
& =-\frac{q_{1}^{2}}{p_{1}^{-}}\left(n^{-}\right)^{\nu_{1}}\left(n^{-}\right)^{\eta}\left(n^{-}\right)^{\sigma}\left[\frac{f_{1 e m} f_{d r m}}{p_{23}^{-}}+\frac{f_{\text {edm }} f_{1 r m}}{q_{2}^{-}}\right], \\
& \Delta_{r 12 e}^{\sigma v_{1} v_{2}+}\left(q_{1}-p_{3}, p_{1}, p_{2}, q_{2}\right) \\
& =\frac{q_{2}^{2}}{p_{1}^{+}}\left(n^{+}\right)^{\nu_{1}}\left(n^{+}\right)^{\nu_{2}}\left(n^{+}\right)^{\sigma}\left[\frac{f_{12 m} f_{\text {erm }}}{p_{12}^{+}}+\frac{f_{2 e m} f_{1 r m}}{p_{2}^{+}}\right], \\
& \Delta_{d r 1 e}^{\rho \sigma v_{1}+}\left(q_{1}, p_{23}, p_{1}, q_{2}\right) \\
& =\frac{q_{2}^{2}}{p_{1}^{+}}\left(n^{+}\right)^{\nu_{1}}\left(n^{+}\right)^{\rho}\left(n^{-}\right)^{\sigma}\left[\frac{f_{d 1 m} f_{e r m}}{p_{23}^{+}}+\frac{f_{e d m} f_{1 r m}}{q_{1}^{+}}\right],
\end{aligned}
$$

with conservation law

$$
q_{1}^{+}=p_{1}^{+}+p_{2}^{+}+p_{3}^{+}, \quad q_{2}^{-}=p_{1}^{-}+p_{2}^{-}+p_{3}^{-} .
$$



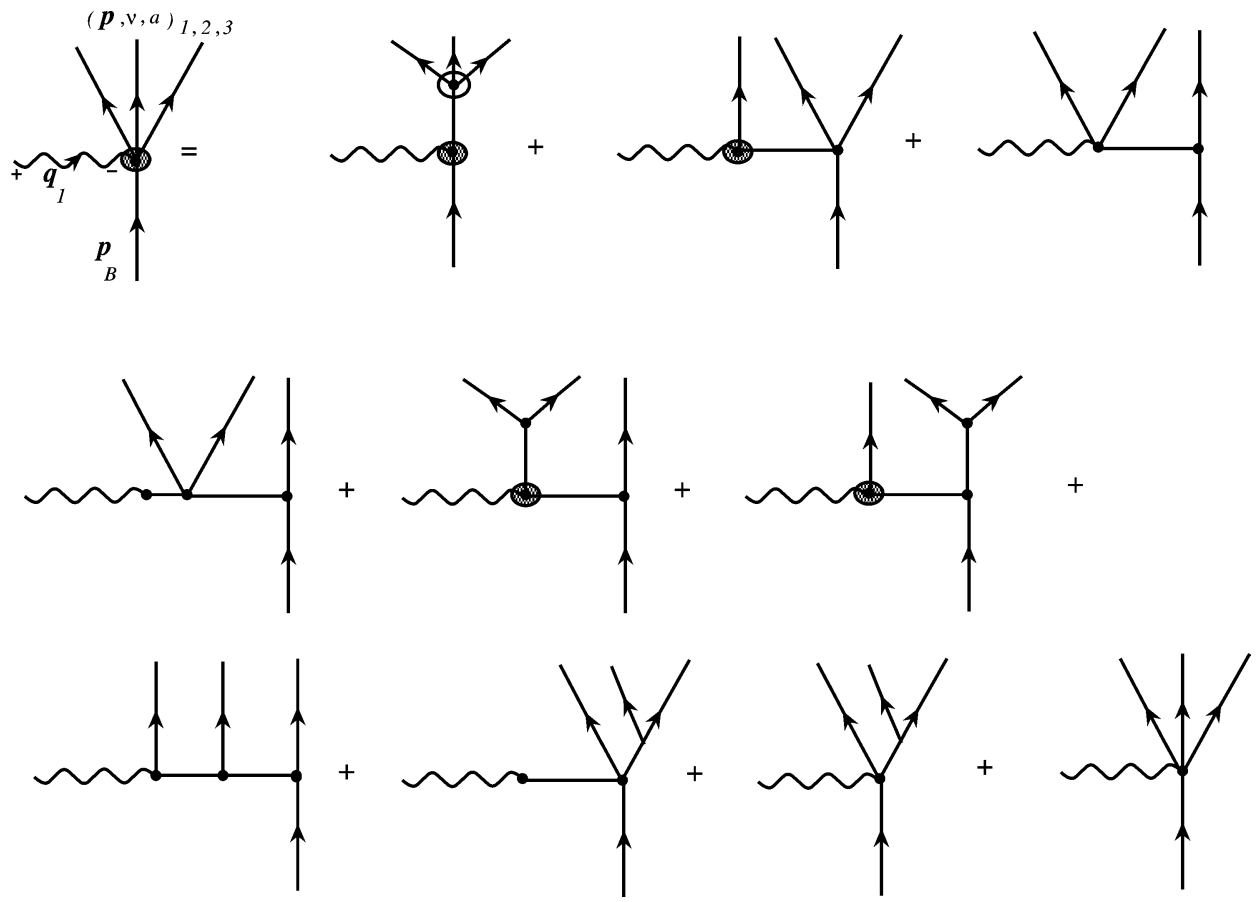

Fig. 10. Margin $P P P P R$ effective vertex: "right" type.

- For the "left" margin effective vertex of the rank-3 (Fig. 9) we have:

$$
\begin{aligned}
\frac{1}{g^{3}} \Gamma_{d 123 e}^{\rho v_{1} v_{2} v_{3}+}\left(P_{A}, p_{1}, p_{2}, p_{3}, q_{2}\right) \\
=\frac{f_{e d r}}{k^{2}} \Gamma^{\rho \sigma+}\left(P_{A}, q_{2}\right) \cdot A_{r 123}^{\sigma v_{1} v_{2} v_{3}}-\left[f_{m d 1} f_{r m 2} f_{e r 3} \frac{1}{\left(P_{A}-p_{1}\right)^{2}\left(p_{3}-q_{2}\right)^{2}}\right. \\
\quad \times \Gamma^{\eta v_{3}+}\left(p_{3}-q_{2}, q_{2}\right) \gamma^{\sigma \nu_{2} \eta}\left(P_{A}-p_{1},-p_{2}, q_{2}-p_{3}\right) \\
\left.\times \gamma^{\rho v_{1} \sigma}\left(P_{A},-p_{1}, p_{1}-P_{A}\right)+5 \text { perm. }\right] \\
-\left[f_{r d m} f_{m 12} f_{e r 3} \frac{1}{p_{12}^{2}\left(p_{3}-q_{2}\right)^{2}} \Gamma^{\eta v_{3}+}\left(p_{3}-q_{2}, q_{2}\right)\right. \\
\left.\times \gamma^{\rho \sigma \eta}\left(P_{A},-p_{12}, q_{2}-p_{3}\right) \gamma^{\sigma v_{1} v_{2}}\left(p_{12},-p_{1},-p_{2}\right)+2 \text { perm. }\right] \\
+\left[f_{m d 1} \frac{1}{\left(p_{1}-P_{A}\right)^{2}} \gamma^{\rho v_{1} \sigma}\left(P_{A},-p_{1}, p_{1}-P_{A}\right)\right. \\
\times\left[-\frac{1}{p_{23}^{2}} \Gamma^{\sigma \eta+}\left(p_{23}-q_{2}, q_{2}\right) \gamma^{\nu_{2} v_{3} \eta}\left(-p_{2},-p_{3}, p_{23}\right) f_{e m r} f_{r 23}\right. \\
\left.\left.+(\Delta+\gamma)_{m 23 e}^{\sigma v_{2} v_{3}+}\left(P_{A}-p_{1}, p_{2}, p_{3}, q_{2}\right)\right]+2 \text { perm. }\right]
\end{aligned}
$$




$$
\begin{aligned}
& +\left[f_{e r 1} \frac{1}{\left(p_{1}-q_{2}\right)^{2}} \Gamma^{\sigma v_{1}+}\left(p_{1}-q_{2}, q_{2}\right) \gamma_{d 23 r}^{\rho v_{2} v_{3} \sigma}+2 \text { perm. }\right] \\
& +\left[\frac{f_{r 23}}{p_{23}^{2}} \gamma^{\nu_{2} v_{3} \eta}\left(-p_{2},-p_{3}, p_{23}\right)(\Delta+\gamma)_{d r 1 e}^{\rho \eta v_{1}+}\left(P_{A}, p_{23}, p_{1}, q_{2}\right)\right. \\
& +2 \text { perm. }] \\
& -\Delta_{d 123 e}^{\rho v_{1} v_{2} v_{3}+}\left(P_{A}, p_{1}, p_{2}, p_{3}, q_{2}\right),
\end{aligned}
$$

where the "left" margin induced vertex, Fig. 3(d) left, reads

$$
\begin{aligned}
& \Delta_{m 23 e}^{\sigma \nu_{2} \nu_{3}+}\left(P_{A}-p_{1}, p_{2}, p_{3}, q_{2}\right) \\
& \quad=\frac{q_{2}^{2}}{p_{23}^{+}}\left(n^{+}\right)^{\sigma}\left(n^{+}\right)^{\nu_{2}}\left(n^{+}\right)^{\nu_{3}}\left(\frac{f_{3 e r} f_{e m r}}{p_{3}^{+}}-\frac{f_{e 2 r} f_{3 m r}}{p_{2}^{+}}\right),
\end{aligned}
$$

and

$$
\begin{aligned}
& \Delta_{d r 1 e}^{\rho \eta v_{1}+}\left(P_{A}, p_{23}, p_{1}, q_{2}\right) \\
& \quad=\frac{q_{2}^{2}}{p_{1}^{+}}\left(n^{+}\right)^{\rho}\left(n^{+}\right)^{v_{1}}\left(n^{+}\right)^{\eta}\left(-\frac{f_{\text {erm }} f_{1 d m}}{p_{23}^{+}}-\frac{f_{r 1 m} f_{e d m}}{P_{A}^{+}}\right),
\end{aligned}
$$

and the conservation law $P_{A}^{+}=p_{1}^{+}+p_{2}^{+}+p_{3}^{+}$is implied.

- The "right" margin effective vertex is (Fig. 10)

$$
\begin{aligned}
& \frac{1}{g^{3}} \Gamma_{e 123 d}^{-v_{1} v_{2} v_{3} \rho}\left(q_{1}, p_{1}, p_{2}, p_{3}, P_{B}\right) \\
& =-\frac{f_{d e r}}{k^{2}} \Gamma^{\rho \sigma-}\left(q_{1}, P_{B}\right) \cdot A_{r 123}^{\sigma v_{1} v_{2} v_{3}} \\
& +\left[f_{m e 1} f_{r m 2} f_{d r 3} \frac{1}{\left(P_{B}-p_{3}\right)^{2}\left(p_{1}-q_{1}\right)^{2}} \Gamma^{\sigma \nu_{1}-}\left(q_{1}, p_{1}-q_{1}\right)\right. \\
& \left.\times \gamma^{\eta \sigma v_{2}}\left(P_{B}-p_{3}, q_{1}-p_{1},-p_{2}\right) \gamma^{\rho \eta v_{3}}\left(P_{B}, p_{3}-P_{B},-p_{3}\right)+5 \text { perm. }\right] \\
& +\left[f_{d r m} f_{m 12} f_{r e 3} \frac{1}{p_{12}^{2}\left(p_{3}-q_{1}\right)^{2}} \Gamma^{\sigma \nu_{3}-}\left(q_{1}, p_{3}-q_{1}\right)\right. \\
& \left.\times \gamma^{\rho \sigma \eta}\left(P_{B}, p_{12}-P_{B},-p_{12}\right) \gamma^{\eta v_{1} v_{2}}\left(p_{12},-p_{1},-p_{2}\right)+2 \text { perm. }\right] \\
& +\left[f_{d r 1} \frac{1}{\left(p_{1}-P_{B}\right)^{2}} \gamma^{\rho \eta v_{1}}\left(P_{B}, p_{1}-P_{B},-p_{1}\right)\right. \\
& \times\left[\frac{f_{\text {rem }} f_{m 23}}{p_{23}^{2}} \Gamma^{\eta \sigma-}\left(q_{1}, p_{23}-q_{1}\right) \gamma^{\sigma \nu_{2} \nu_{3}}\left(p_{23},-p_{2},-p_{3}\right)\right. \\
& \left.\left.+(\Delta+\gamma)_{e 23 r}^{-v_{2} v_{3} \eta}\left(q_{1}, p_{2}, p_{3}, P_{B}-p_{1}\right)\right]+2 \text { perm. }\right] \\
& -\left[f_{m e 1} \frac{1}{\left(p_{1}-q_{1}\right)^{2}} \Gamma^{\sigma v_{1}-}\left(q_{1}, p_{1}-q_{1}\right) \gamma_{m 23 d}^{\sigma v_{2} \nu_{3} \rho}+2 \text { perm. }\right]
\end{aligned}
$$




$$
\begin{aligned}
& +\left[\frac{f_{m 23}}{p_{23}^{2}} \gamma^{\sigma v_{2} v_{3}}\left(p_{23},-p_{2},-p_{3}\right)(\Delta+\gamma)_{e m 1 d}^{-\sigma v_{1} \rho}\left(q_{1}, p_{23}, p_{1}, P_{B}\right)\right. \\
& +2 \text { perm. }] \\
& -\Delta_{e 123 d}^{-v_{1} v_{2} \nu_{3} \rho}\left(q_{1}, p_{1}, p_{2}, p_{3}, P_{B}\right)
\end{aligned}
$$

with the induced vertex, Fig. 3(d) right:

$$
\begin{aligned}
& \Delta_{e 23 r}^{-v_{2} \nu_{3} \eta}\left(q_{1}, p_{2}, p_{3}, P_{B}-p_{1}\right) \\
& \quad=\frac{q_{1}^{2}}{p_{23}^{-}}\left(n^{-}\right)^{\nu_{2}}\left(n^{-}\right)^{\nu_{3}}\left(n^{-}\right)^{\eta}\left[\frac{f_{3 e m} f_{2 r m}}{p_{3}^{-}}-\frac{f_{e 2 m} f_{3 r m}}{p_{2}^{-}}\right],
\end{aligned}
$$

and

$$
\begin{aligned}
& \Delta_{e m 1 d}^{-\sigma \nu_{1} \rho}\left(q_{1}, p_{23}, p_{1}, P_{B}\right) \\
& \quad=\frac{q_{1}^{2}}{p_{1}^{-}}\left(n^{-}\right)^{\nu_{1}}\left(n^{-}\right)^{\rho}\left(n^{-}\right)^{\sigma}\left[\frac{f_{e d r} f_{1 m r}}{P_{B}^{-}}-\frac{f_{d 1 r} f_{e m r}}{p_{23}^{-}}\right],
\end{aligned}
$$

and the conservation law $P_{B}^{-}=p_{1}^{-}+p_{2}^{-}+p_{3}^{-}$.

Here by term "perm." we imply all the terms obtained from the explicitly written ones by simultaneous permutations of the momenta, color and Lorentz indices. Like in the previous cases, the gauge invariance and Bose-symmetry properties are fulfilled explicitly. Some useful relations which can be used are presented in Appendix A.

The "left" induced vertex of the rank-3 has a form

$$
\begin{aligned}
& \Delta_{d 123 e}^{\rho v_{1} \nu_{2} \nu_{3}+}\left(q_{1}, p_{1}, p_{2}, p_{3}, q_{2}\right) \\
& =q_{2}^{2}\left(n^{+}\right)^{\rho}\left(n^{+}\right)^{\nu_{1}}\left(n^{+}\right)^{\nu_{2}}\left(n^{+}\right)^{\nu_{3}} \frac{D^{+}\left(q_{1}, d ; p_{1}, a_{1} ; p_{2}, a_{2} ; p_{3}, a_{3} ; q_{2}, e\right)}{p_{1}^{+}},
\end{aligned}
$$

where

$$
\begin{aligned}
D^{+} & \left(q_{1}, d ; p_{1}, a_{1} ; p_{2}, a_{2} ; p_{3}, a_{3} ; q_{2}, e\right) \\
& =\frac{1}{p_{23}^{+}}\left[\frac{\alpha_{1}}{p_{2}^{+}}-\frac{\alpha_{2}}{p_{3}^{+}}\right]+\frac{1}{p_{12}^{+}}\left[\frac{\alpha_{3}}{q_{1}^{+}}+\frac{\alpha_{4}}{p_{3}^{+}}\right]+\frac{1}{p_{13}^{+}}\left[\frac{\alpha_{5}}{q_{1}^{+}}+\frac{\alpha_{6}}{p_{2}^{+}}\right],
\end{aligned}
$$

and

$$
\begin{array}{lll}
\alpha_{1}=f_{m d 1} f_{e 2 r} f_{3 m r} ; & \alpha_{2}=f_{m d 1} f_{3 e r} f_{2 m r} ; & \alpha_{3}=f_{m 12} f_{d e r} f_{3 m r} \\
\alpha_{4}=f_{m 12} f_{e 3 r} f_{d m r} ; & \alpha_{1}=f_{m 13} f_{d e r} f_{2 m r} ; & \alpha_{6}=f_{m 13} f_{e 2 r} f_{d m r} .
\end{array}
$$

For the "right" induced vertex of the rank-3 (Fig. 3(g) right) we have

$$
\begin{aligned}
& \Delta_{d a_{1} a_{2} a_{3} e}^{-v_{1} \nu_{2} \nu_{3} \rho}\left(q_{1}, p_{1}, p_{2}, p_{3}, q_{2}\right) \\
& \quad=q_{1}^{2}\left(n^{-}\right)^{\nu_{1}}\left(n^{-}\right)^{\nu_{2}}\left(n^{-}\right)^{\nu_{3}}\left(n^{-}\right)^{\rho} \frac{D^{-}\left(q_{1}, d ; p_{1}, a_{1} ; p_{2}, a_{2} ; p_{3}, a_{3} ; q_{2}, e\right)}{p_{1}^{-}},
\end{aligned}
$$


where

$$
\begin{aligned}
D^{-} & \left(q_{1}, d ; p_{1}, a_{1} ; p_{2}, a_{2} ; p_{3}, a_{3} ; q_{2}, e\right) \\
& =\frac{1}{p_{23}^{-}}\left(\frac{\gamma_{1}}{p_{3}^{-}}-\frac{\gamma_{2}}{p_{2}^{-}}\right)-\frac{1}{p_{12}^{-}}\left(\frac{\gamma_{3}}{q_{2}^{-}}+\frac{\gamma_{4}}{p_{3}^{-}}\right)-\frac{1}{p_{13}^{-}}\left(\frac{\gamma_{5}}{q_{2}^{-}}+\frac{\gamma_{6}}{p_{2}^{-}}\right),
\end{aligned}
$$

and

$$
\begin{array}{lll}
\gamma_{1}=f_{m 1 e} f_{3 d r} f_{2 m r} ; & \gamma_{2}=f_{m 1 e} f_{d 2 r} f_{3 m r} ; & \gamma_{3}=f_{m 12} f_{d e r} f_{3 m r} \\
\gamma_{4}=f_{m 12} f_{3 d r} f_{\text {emr }} ; & \gamma_{5}=f_{m 13} f_{d e r} f_{2 m r} ; & \gamma_{6}=f_{m 13} f_{2 d r} f_{e m r} .
\end{array}
$$

The Bose-symmetry can be proven similarly to the case of the "right" induced vertex of the rank-4, if one takes into account the relation

$$
\begin{aligned}
& \Delta_{e a_{1} a_{2} a_{3} d}^{-v_{1} v_{2} v_{3} \rho}\left(q_{1}, p_{1}, p_{2}, p_{3}, q_{2}\right) \\
& \quad=\left.\Delta_{d a_{1} a_{2} a_{3} e}^{\rho v_{1} v_{2} v_{3}}\left(q_{1}, p_{1}, p_{2}, p_{3}, q_{2}\right)\right|_{p_{i}^{+} \rightarrow p_{i}^{-} ; d \rightarrow e ; e \rightarrow d ; q_{1}^{+} \rightarrow q_{2}^{-}} .
\end{aligned}
$$

Besides this, let us note that the margin induced vertices of the rank-3 are connected with the central ones:

$$
\begin{aligned}
& \Delta_{d a_{1} a_{2} a_{3} e}^{-v_{1} v_{2} v_{3} \rho}\left(q_{1}, p_{1}, p_{2}, p_{3}, P_{B}\right)=\left.\Delta_{e a_{1} a_{2} a_{3} d}^{-v_{1} v_{2} v_{3} \rho}\left(q_{1}, p_{1}, p_{2}, p_{3}, q_{2}\right)\right|_{q_{2} \rightarrow P_{B} ; d \rightarrow e ; e \rightarrow d}, \\
& \Delta_{e a_{1} a_{2} a_{3} d}^{\rho v_{1} \nu_{2} v_{3}+}\left(P_{A}, p_{1}, p_{2}, p_{3}, q_{2}\right)=\left.\Delta_{e a_{1} a_{2} a_{3} d}^{\rho v_{1} v_{2} v_{3}+}\left(q_{1}, p_{1}, p_{2}, p_{3}, q_{2}\right)\right|_{q_{1} \rightarrow P_{A}} .
\end{aligned}
$$

The effective 4-gluon vertex $A$ entering to $R R P P P$ and $R P P P P$ vertices reads

$$
\begin{aligned}
A_{a_{1} a_{2} a_{3} r}^{\nu_{1} \nu_{2} \nu_{3} \sigma}= & \tau_{1}\left[g^{\nu_{1} \sigma} g^{\nu_{2} \nu_{3}}-g^{\nu_{1} \nu_{3}} g^{\nu_{2} \sigma}\right]+\tau_{2}\left[g^{\nu_{1} \nu_{2}} g^{\nu_{3} \sigma}-g^{\nu_{1} \sigma} g^{\nu_{2} \nu_{3}}\right] \\
& +\tau_{3}\left[g^{\nu_{1} \nu_{3}} g^{\nu_{2} \sigma}-g^{\nu_{1} \nu_{2}} g^{\nu_{3} \sigma}\right] \\
& +\frac{\tau_{3}}{p_{23}^{2}} \gamma^{\sigma \nu_{1} \rho}\left(k,-p_{1},-p_{2}-p_{3}\right) \gamma^{\rho \nu_{2} \nu_{3}}\left(p_{2}+p_{3},-p_{2},-p_{3}\right) \\
& +\frac{\tau_{2}}{p_{13}^{2}} \gamma^{\sigma \rho \nu_{2}}\left(k,-p_{1}-p_{3},-p_{2}\right) \gamma^{\rho \nu_{1} \nu_{3}}\left(p_{1}+p_{3},-p_{1},-p_{3}\right) \\
& -\frac{\tau_{1}}{p_{12}^{2}} \gamma^{\sigma \rho \nu_{3}}\left(k,-p_{1}-p_{2},-p_{3}\right) \gamma^{\rho \nu_{1} \nu_{2}}\left(p_{1}+p_{2},-p_{1},-p_{2}\right),
\end{aligned}
$$

where

$$
\tau_{1}=f_{12 m} f_{3 r m}, \quad \tau_{2}=f_{31 m} f_{2 r m}, \quad \tau_{3}=f_{23 m} f_{1 r m}, \quad \tau_{1}+\tau_{2}+\tau_{3}=0 .
$$

\section{Cross sections for jet production in QMRK}

The typical process of the $n$-jet production in the quasi-multi-Regge kinematics is presented graphically at Fig. 1. Within this regime, one can use the Sudakov decomposition

$$
q_{i}=\alpha_{i} P_{B}+\beta_{i} P_{A}+\boldsymbol{q}_{i \perp}, \quad \boldsymbol{q}_{i \perp} \cdot P_{A}=\boldsymbol{q}_{i \perp} \cdot P_{B}=0, \quad d^{4} q_{i}=\frac{s}{2} d \alpha_{i} d \beta_{i} d^{2} \boldsymbol{q}_{i},
$$


and the invariant masses of jets:

$$
\begin{aligned}
& \left(P_{A}-q_{1}\right)^{2}=M_{1}^{2} \approx-s \alpha_{1}, \\
& \left(q_{1}-q_{2}\right)^{2}=M_{2}^{2} \approx-s \alpha_{2} \beta_{1}, \\
& \vdots \\
& \left(q_{n-2}-q_{n-1}\right)^{2}=M_{n-1}^{2} \approx-s \alpha_{n-1} \beta_{n-2}, \\
& \left(q_{n-1}+P_{B}\right)^{2}=M_{n}^{2} \approx s \beta_{n-1},
\end{aligned}
$$

by virtue of the ordering valid in the QMRK:

$$
\begin{aligned}
& \alpha_{1} \ll \alpha_{2} \ll \cdots \ll \alpha_{n}, \quad \beta_{n-1} \ll \beta_{n-2} \ll \cdots \ll \beta_{1}, \quad M_{i}^{2} \sim M^{2} \ll s, \\
& s_{1}=\left(P_{A}-q_{2}\right)^{2} \sim s_{2}=\left(q_{1}-q_{3}\right)^{2} \sim \cdots \sim s_{n-1}=\left(q_{n-2}+P_{B}\right)^{2} \gg M^{2} .
\end{aligned}
$$

Here $M_{i}$ is the effective mass of the $i$ th jet. These quantities are related as $\prod_{1}^{n-1} s_{i}=$ $s \prod_{2}^{n-1} M_{i}^{2}$.

The differential cross section of the $n$ jet production is given by

$$
d \sigma^{2 \rightarrow n_{\mathrm{jet}}}=\frac{1}{8 s}\left|\mathcal{M}^{2 \rightarrow n}\right|^{2} d \Gamma_{n}^{(\mathrm{jet})} .
$$

The phase volume for the $n$ jets containing $n_{i}, i=1, \ldots, n$, produced particles in each jet can be written as

$$
\begin{aligned}
d \Gamma_{n}^{(\mathrm{jet})} & =\frac{(2 \pi)^{4}}{s \cdot 2^{n-1}} \prod_{i=1}^{n-1} d^{2} \boldsymbol{q}_{i} \prod_{i=1}^{n} d \phi_{i} \int_{\frac{M^{2}}{s}}^{1} \frac{d \beta_{1}}{\beta_{1}} \int_{\beta_{1}}^{1} \frac{d \beta_{2}}{\beta_{2}} \cdots \int_{\beta_{n-3}}^{1} \frac{d \beta_{n-2}}{\beta_{n-2}} \\
& =\frac{(2 \pi)^{4}}{s \cdot 2^{n-1}} \prod_{i=1}^{n-1} d^{2} \boldsymbol{q}_{i} \prod_{i=1}^{n} d \phi_{i} \cdot \frac{\left(\ln \frac{s}{M^{2}}\right)^{n-2}}{(n-2) !},
\end{aligned}
$$

where

$$
\begin{aligned}
& d \phi_{1}=d M_{1}^{2} d \Gamma_{1}(2 \pi)^{4} \delta^{(4)}\left(P_{A}-q_{1}-\sum_{j=1}^{n_{1}} p_{j}^{(1)}\right), \\
& d \phi_{i}=d M_{i}^{2} d \Gamma_{i}(2 \pi)^{4} \delta^{(4)}\left(q_{i-1}-q_{i}-\sum_{j=1}^{n_{2}} p_{j}^{(i)}\right), \quad i=2, \ldots, n-1, \\
& d \phi_{n}=d M_{n}^{2} d \Gamma_{n}(2 \pi)^{4} \delta^{(4)}\left(q_{n-1}+P_{B}-\sum_{j=1}^{n_{n}} p_{j}^{(n)}\right),
\end{aligned}
$$

and

$$
d \Gamma_{i}=\prod_{j=1}^{\left(n_{i}\right)} \frac{d^{3} p_{j}^{(i)}}{2 \varepsilon_{j}(2 \pi)^{3}} .
$$


Therefore, taking into account that the amplitude for $2 \rightarrow n$ production is given by

$$
\mathcal{M}^{2 \rightarrow n}=2^{-n+1} s \cdot g^{n} V_{A} V_{B}\left[\prod_{i=2}^{n-1} \frac{V_{i}^{i_{1} \ldots i_{n_{i}}}\left(q_{i}, q_{i+1}\right)}{q_{i}^{2}}\right],
$$

where $V_{i}\left(q_{i}, q_{i+1}\right)$ are the $R R P^{n_{i}}$-vertices for $n_{i}$-particle production in $i$ th jet. The margin vertices defined as

$$
V_{A}=\frac{1}{P_{A}^{+}} \Gamma_{\|}^{\rho v_{(i)}-} e_{\rho}\left(p_{A}\right) \prod e_{v_{i}}\left(p_{i}^{(1)}\right), \quad V_{B}=\frac{1}{P_{B}^{-}} \Gamma_{\|}^{\eta v_{(j)}+} e_{\eta}\left(p_{B}\right) \prod e_{v_{j}}\left(p_{j}^{(n)}\right) .
$$

For the case when a jet consists from one particle, we have omitting the color factor:

$$
V_{A}=g \frac{\Gamma^{\sigma \eta+}\left(P_{A}, q_{2}\right)}{P_{A}^{+}} \cdot e_{\sigma}\left(P_{A}\right) e_{\eta}\left(P_{A^{\prime}}\right) ; \quad \sum\left(V_{A}\right)^{2}=8 g^{2} .
$$

The differential cross section reads:

$$
\begin{aligned}
d \sigma^{2 \rightarrow n}= & R(s) \alpha_{s}^{n} 2^{-5 n+4} \pi^{-2 n+3} \cdot V_{A}^{2} V_{B}^{2} \\
& \times \prod_{i=2}^{n-1}\left(V_{i}\right)^{2} \cdot \frac{\prod_{i=1}^{n-1} d^{2} \boldsymbol{q}_{i}}{\left(\prod_{i=1}^{n-1} \boldsymbol{q}_{i}^{2}\right)^{2}} \cdot \prod_{i=1}^{n} d \phi_{i} \frac{\left(\ln s / M^{2}\right)^{n-2}}{(n-2) !},
\end{aligned}
$$

where the intermediate gluons reggeization factor is included:

$$
R(s)=\prod_{i=1}^{n-1}\left(\frac{s_{i}}{M^{2}}\right)^{2\left(\omega\left(q_{i}^{2}\right)-1\right)}
$$

with $M$ is some averaged value of jet invariant mass and $\omega\left(q^{2}\right), \omega(0)=1$ is the gluon Regge trajectory.

\section{Discussions and conclusions}

The primary purpose of our investigation is to give a systematic self-consistent approach to derivation of the Feynman rules directly from the effective reggeon-particle action, and, what is essential, to present the results in a most convenient form for numerical simulations. We hope that our results will provide a practical help in writing relevant computer codes for phenomenological needs. In this work, we present the explicit expressions for effective vertices of the types $P R, R P, R P P, R R P, R R P P, R P P P, R R P P P, R P P P P$ derived directly within the framework of the effective Regge theory developed in the paper by one of us (L.N.L) [8]. Some of these vertices were obtained by means of different methods in the Refs. [9,20,24,27], while the two last vertices ( $R R P P P$ and $R P P P P$ ) were not considered in literature up to now. All presented vertices satisfy the Bose-symmetry and gauge invariance conditions. Moreover, we made sure that the vertices of higher orders can be constructed, step by step, using the known ones given above. 
The "non-linear" vertices of type $R R R P$ with number of reggeons exceeding two can be constructed as well. We will not consider them here. The program of fitting the experimental data in the QMRK using the effective vertices given above can be realized say for extracting the information about the gluon reggeon parameters.

\section{Acknowledgements}

The work is partially supported by INTAS (Grant No. 00-00-366), RFBR (Grants Nos. 04-02-16445, 03-02-17077), and Russian Federation President's Grant 1450-2003-2. We thank V.S. Fadin, J. Bartels, R. Kirschner, B. Nicolescu, G. Salam, N.N. Nikolaev, G.P. Vacca, M.A. Braun, V. Saleev, and others for helpful discussions. We are grateful to V. Bytev for help.

\section{Appendix A}

For convenience, we collect here several useful relations for vertices, which were derived and used in this work (here it is assumed that $k=p_{1}+p_{2}+p_{3}$ ).

Effective 4-gluon vertex entering $R R P P P$ and $R P P P P$ has a property:

$$
p_{1 \nu_{1}} A_{r 123}^{\sigma \nu_{1} \nu_{2} \nu_{3}}=k^{2}\left[\frac{\tau_{1}}{p_{12}^{2}} p_{1}^{\nu_{2}} g^{\sigma \nu_{3}}-\frac{\tau_{2}}{p_{23}^{2}} \gamma^{\nu_{2} \nu_{3} \sigma}\left(-p_{2},-p_{3}, p_{23}\right)-\frac{\tau_{3}}{p_{13}^{2}} p_{1}^{\nu_{3}} g^{\sigma \nu_{2}}\right],
$$

with

$$
\tau_{1}=f_{12 m} f_{3 r m}, \quad \tau_{2}=f_{1 r m} f_{23 m}, \quad \tau_{3}=f_{13 m} f_{r 2 m}, \quad \sum \tau_{i}=0 .
$$

Let us demonstrate the Bose-symmetry of induced $\Delta_{d 123 e}^{\rho v_{1} \nu_{2} v_{3}+}\left(q_{1}, p_{1}, p_{2}, p_{3}, q_{2}\right)$ vertex. Really we have:

$$
\begin{aligned}
& D^{+}\left(q_{1}, d ; p_{1}, a_{1} ; p_{2}, a_{2} ; p_{3}, a_{3} ; q_{2}, e\right)=D^{+}\left(q_{1}, d ; p_{1}, a_{1} ; p_{3}, a_{3} ; p_{2}, a_{2} ; q_{2}, e\right) \\
& \frac{D^{+}\left(q_{1}, d ; p_{1}, a_{1} ; p_{2}, a_{2} ; p_{3}, a_{3} ; q_{2}, e\right)}{p_{1}^{+}}=\frac{D^{+}\left(q_{1}, d ; p_{2}, a_{2} ; p_{1}, a_{1} ; p_{3}, a_{3} ; q_{2}, e\right)}{p_{2}^{+}} .
\end{aligned}
$$

The first relation is obvious by construction. To show the validity of the second one, let us note that the expression

$$
\frac{1}{p_{2}^{+}}\left[\frac{1}{p_{13}^{+}}\left(\frac{\beta_{1}}{p_{1}^{+}}-\frac{\beta_{2}}{p_{3}^{+}}\right)+\frac{1}{p_{12}^{+}}\left(\frac{\beta_{3}}{q_{1}^{+}}+\frac{\beta_{4}}{p_{3}^{+}}\right)+\frac{1}{p_{23}^{+}}\left(\frac{\beta_{5}}{q_{1}^{+}}+\frac{\beta_{6}}{p_{1}^{+}}\right)\right],
$$

where

$$
\begin{array}{lll}
\beta_{1}=f_{m d 2} f_{e 1 r} f_{3 m r} ; & \beta_{2}=f_{m d 2} f_{3 e r} f_{1 m r} ; & \beta_{3}=f_{m 21} f_{d e r} f_{3 m r} \\
\beta_{4}=f_{m 21} f_{e 3 r} f_{d m r} ; & \beta_{5}=f_{m 23} f_{d e r} f_{1 m r} ; & \beta_{6}=f_{m 23} f_{e 1 r} f_{d m r}
\end{array}
$$


which is indeed the r.h.s. of the second equation, can be rearranged using the conservation law $q_{1}^{+}=p_{1}^{+}+p_{2}^{+}+p_{3}^{+}$and the relations

$$
\begin{aligned}
& \beta_{1}=-\alpha_{2}+\alpha_{4}+\alpha_{5}+\alpha_{6} ; \quad \beta_{2}=\alpha_{2}-\alpha_{4} ; \quad \beta_{3}=-\alpha_{3} ; \\
& \beta_{4}=-\alpha_{4} ; \quad \beta_{5}=-\alpha_{3}+\alpha_{5} ; \quad \beta_{6}=\alpha_{1}+\alpha_{2}+\alpha_{3}-\alpha_{5}
\end{aligned}
$$

giving the 1.h.s. of Eq. (A.2).

For testing the gauge properties of margin vertices some identities can be used:

$$
\begin{aligned}
& x_{1} \gamma^{\nu_{2} \nu_{3} \rho}\left(-p_{2},-p_{3}, p_{23}\right)+x_{2} \gamma^{\nu_{3} \rho \nu_{2}}\left(p_{2}-P_{A}, P_{A},-p_{2}\right) \\
& \quad-x_{3} \gamma^{\nu_{2} \rho \nu_{3}}\left(p_{3}-P_{A}, P_{A},-p_{3}\right)+\gamma_{d 23 r}^{\rho \nu_{2} \nu_{3} \sigma}\left(p_{1}-q_{2}\right)_{\sigma}=0,
\end{aligned}
$$

with

$$
x_{1}=f_{32 m} f_{d r m}, \quad x_{2}=f_{2 d m} f_{3 r m}, \quad x_{3}=f_{d 3 m} f_{2 r m}, \quad x_{1}+x_{2}+x_{3}=0,
$$

and, besides this:

$$
\begin{aligned}
& f_{1 e m} f_{d 2 r} f_{m 3 r}-f_{13 r} f_{2 d m} f_{e r m}-f_{r d 1} f_{r 2 m} f_{e 3 m}+f_{12 r} f_{e 3 m} f_{d r m}=0 \\
& f_{m 1 e} \gamma_{d 23 m}^{-\nu_{2} \nu_{3}+}+f_{m 1 d} \gamma_{m 23 e}^{-\nu_{2} \nu_{3}+}+f_{m 12} \gamma_{d m 3 m}^{-\nu_{2} \nu_{3}+}+f_{m 13} \gamma_{d 2 m e}^{-\nu_{2} \nu_{3}+}=0 .
\end{aligned}
$$

\section{References}

[1] V.S. Fadin, E.A. Kuraev, L.N. Lipatov, Phys. Lett. B 60 (1975) 50;

E.A. Kuraev, L.N. Lipatov, V.S. Fadin, Zh. Eksp. Teor. Fiz. 71 (1976) 840, Sov. Phys. JETP 44 (1976) 443; E.A. Kuraev, L.N. Lipatov, V.S. Fadin, Zh. Eksp. Teor. Fiz. 72 (1977) 377, Sov. Phys. JETP 45 (1977) 199; I.I. Balitsky, L.N. Lipatov, Yad. Fiz. 28 (1978) 1597, Sov. J. Nucl. Phys. 28 (1978) 822.

[2] V.N. Gribov, L.N. Lipatov, Sov. J. Nucl. Phys. 18 (1972) 438; V.N. Gribov, L.N. Lipatov, Sov. J. Nucl. Phys. 18 (1972) 675;

L.N. Lipatov, Sov. J. Nucl. Phys. 20 (1975) 93;

G. Altarelli, G. Parisi, Nucl. Phys. B 126 (1977) 298;

Yu.L. Dokshitzer, Sov. Phys. JETP 46 (1977) 641.

[3] M. Derrick, et al., ZEUS Collaboration, Phys. Lett. B 345 (1995) 576;

C. Adloff, et al., H1 Collaboration, Eur. Phys. J. C 21 (2001) 33;

S. Chekanov, et al., ZEUS Collaboration, Eur. Phys. J. C 21 (2001) 443.

[4] H. Cheng, T.T. Wu, Expanding Protons: Scattering at High Energies, MIT Press, Cambridge, 1987.

[5] J. Bartels, Nucl. Phys. B 175 (1980) 365;

J. Kwiecinski, M. Praszalowicz, Phys. Lett. B 94 (1980) 413.

[6] L.N. Lipatov, High energy asymptotics of multi-colour QCD and exactly solvable lattice models, hepth/9311037, Padova, preprint DFPD/93/TH, unpublished.

[7] L.N. Lipatov, Nucl. Phys. B 365 (1991) 614;

R. Kirschner, L.N. Lipatov, L. Szymanowski, Nucl. Phys. B 425 (1994) 579.

[8] L.N. Lipatov, Nucl. Phys. B 452 (1995) 369.

[9] L.N. Lipatov, Phys. Rep. 286 (1997) 131.

[10] O. Nachtmann, Ann. Phys. 209 (1991) 436.

[11] H. Verlinde, E. Verlinde, QCD at high energies and two-dimensional field theory, Preprint PUPT 1319, IASSNS-HEP 92/30 (1993), hep-th/9302104.

[12] I.Ya. Aref'eva, Large $N$ QCD at high energies as two-dimensional field theory, Phys. Lett. B 328 (1994) 411, hep-th/9306014. 
[13] I. Balitsky, Nucl. Phys. B 463 (1996) 99;

I. Balitsky, Phys. Rev. D 60 (1999) 014020.

[14] J. Jalilian-Marian, A. Kovner, A. Leonidov, H. Weigert, Nucl. Phys. B 504 (1997) 415;

J. Jalilian-Marian, A. Kovner, A. Leonidov, H. Weigert, Phys. Rev. D 59 (1999) 014014;

E. Iancu, A. Leonidov, L. McLerran, Nucl. Phys. A 692 (2001) 583;

E. Ferreiro, E. Iancu, A. Leonidov, L. McLerran, Nucl. Phys. A 703 (2002) 489.

[15] Y.V. Kovchegov, Phys. Rev. D 60 (1999) 034008;

Y.V. Kovchegov, Phys. Rev. D 61 (2000) 074018.

[16] N.N. Nikolaev, B.G. Zakharov, Phys. Lett. B 327 (1994) 149;

N.N. Nikolaev, B.G. Zakharov, Phys. Lett. B 327 (1994) 157;

A.N. Mueller, Nucl. Phys. B 415 (1994) 377.

[17] J. Bartels, L.N. Lipatov, G.P. Vacca, Nucl. Phys. B 706 (2005) 391.

[18] V.T. Kim, G.B. Pivovarov, Phys. Rev. Lett. 79 (1997) 809.

[19] L.N. Lipatov, Nucl. Phys. B 309 (1988) 379.

[20] L.N. Lipatov, V.S. Fadin, JETP Lett. 49 (1989) 352;

L.N. Lipatov, V.S. Fadin, Yad. Fiz. 50 (1989) 1141.

[21] V.S. Fadin, L.N. Lipatov, Phys. Lett. B 429 (1998) 127.

[22] M. Ciafaloni, G. Camici, Phys. Lett. B 430 (1998) 349.

[23] S.J. Brodsky, V.S. Fadin, V.T. Kim, L.N. Lipatov, G.B. Pivovarov, JETP Lett. 70 (1999) 155; S.J. Brodsky, V.S. Fadin, V.T. Kim, L.N. Lipatov, G.B. Pivovarov, JETP Lett. 76 (2002) 249.

[24] V.S. Fadin, L.N. Lipatov, Nucl. Phys. B 477 (1996) 767.

[25] G. Altarelli, R.D. Ball, S. Forte, Nucl. Phys. B 674 (2003) 459, hep-ph/0407153.

[26] M. Ciafaloni, D. Colferai, G.P. Salam, A.M. Stasto, Phys. Lett. B 587 (2004) 87.

[27] A. Leonidov, D. Ostrovsky, Phys. Rev. D 62 (2000) 094009.

[28] J. Schwarz, Phys. Rep. 89 (1982) 223;

E.N. Antonov, Preprint PNPI-1808, 1992. 